\title{
Numerical Simulation of Transpiration Cooling through Porous Material
}

\author{
Wolfgang Dahmen ${ }^{1}$, Thomas Gotzen ${ }^{1}$ and \\ Siegfried Müller ${ }^{1 *}$
}

Bericht Nr. 374

September 2013

Key words: Transpiration cooling, porous media flow, Darcy-Forchheimer equation, coupled finite element - finite volume schemes

AMS Subject Classifications: 76Sxx, 76N15, 65N30

Institut für Geometrie und Praktische Mathematik RWTH Aachen

Templergraben 55, D-52056 Aachen (Germany)

1* Correspondence to: Institut für Geometrie und Praktische Mathematik, RWTH Aachen University, Templergraben 55, 52056 Aachen. E-mail: mueller@igpm.rwth-aachen.de

Contract/grant sponsor: Financial support has been provided by the German Research Foundation (Deutsche Forschungsgemeinschaft - DFG) in the framework of the Sonderforschungsbereich Transregio 40 and through the Graduate School AICES (GSC 111). 


\title{
Numerical simulation of transpiration cooling through porous material
}

\author{
W. Dahmen ${ }^{1}$, T. Gotzen ${ }^{1}$ and S. Müller ${ }^{1 *}$ \\ ${ }^{1}$ Institut für Geometrie und Praktische Mathematik, RWTH Aachen University, Templergraben 55, 52056 Aachen
}

\begin{abstract}
SUMMARY
Transpiration cooling using ceramic matrix composite (CMC) materials is an innovative concept for cooling rocket thrust chambers. The coolant (air) is driven through the porous material by a pressure difference between the coolant reservoir and the turbulent hot gas flow. The effectiveness of such cooling strategies relies on a proper choice of the involved process parameters such as injection pressure, blowing ratios, material structure parameters, to name only a few. In view of the limited experimental access to the subtle processes occurring at the interface between hot gas flow and porous medium, reliable and accurate simulations become an increasingly important design tool. In order to facilitate such numerical simulations for a carbon/carbon material mounted in the side wall of a hot gas channel that are able to capture a spatially varying interplay between the hot gas flow and the coolant at the interface, we formulate a two dimensional model for the porous medium flow of Darcy-Forchheimer type. A finite element solver for the corresponding porous media flow is presented and coupled with a finite volume solver for the compressible Reynolds averaged Navier-Stokes equations. The results at Mach number $M a=0.5$ and hot gas temperature $T_{h g}=540 \mathrm{~K}$ for different blowing ratios are compared with experiments.
\end{abstract}

KEY WORDS: Transpiration cooling, porous media flow, Darcy-Forchheimer equation, coupled finite element-, finite volume schemes, numerical tests

\section{INTRODUCTION}

Significantly increasing the efficiency of future space transportation systems relies crucially on allowing higher payloads since more powerful rocket engines inevitably entail higher thermal loads on the structure. An improved performance of the rocket engines should not be accomplished though at the expense of an increased weight of the rocket itself. This calls for new cooling technologies, for example, in the combustion chamber of the rocket engine where high thermal loads up to $100 \mathrm{MW} / \mathrm{m}^{2}$ occur [1] are encountered. Innovative cooling methods that are able to deal with such loads are currently under investigation. Needless to stress, that the reliability of such a cooling

\footnotetext{
*Correspondence to: Institut für Geometrie und Praktische Mathematik, RWTH Aachen University, Templergraben 55, 52056 Aachen. E-mail: mueller@igpm.rwth-aachen.de

Contract/grant sponsor: Financial support has been provided by the German Research Foundation (Deutsche Forschungsgemeinschaft - DFG) in the framework of the Sonderforschungsbereich Transregio 40 and through the Graduate School AICES (GSC 111).
} 
concept is essential since a failure of any of its components may lead to the total loss of the mission. Therefore, a detailed understanding of all involved physical effects is indispensable in the design of a new cooling technology.

Existing cooling technologies for space transportation systems can be divided into two main categories, namely passive and active cooling techniques. The most prominent example for a passive cooling techniques is a heat shield, where the structure is protected against high thermal loads by coating it with special materials. In contrast, active systems, which typically employ some sort of cooling fluid, are adjustable during the flight and may therefore offer more favorable efficiency/weight balances.

Active cooling techniques like film cooling have become a common technology, for example, in gas turbines. Accordingly, they have been widely investigated in subsonic, turbulent flow regimes. These techniques are currently being considered also for combustion chambers, where the flow remains subsonic but much higher pressures occur. New basic research is necessary for understanding the physical effects which come along with these new challenges. Experimental studies provide important contributions but are subject to inherent limitations. Often, e.g., due to limited measurement techniques, only a few simultaneous measurements of some quantities are possible which then fail to give any causal insight in the flow field. Furthermore, experiments can become very expensive. Therefore, experiments have to be complemented by numerical simulations which can provide a much more substantial insight into the whole flow field.

\subsection{State of the art}

A very promising cooling strategy for combustion chambers is transpiration cooling, where the coolant is injected through a porous material used as a liner in the combustion chamber. The availability of lightweight materials with both high thermal resistance and suitable porosity has greatly stimulated the investigation of transpiration cooling through experiments. System studies verifying that transpiration cooling can yield better performance than regenerative cooling techniques for rocket thrust chambers can be found in Herbertz [12] and Greuel et al. [13]. In addition, the latter present an experimental-numerical approach and conclude that for a blowing ratio interval $F \in[0.45 \%, 0.7 \%]$ transpiration cooling outperforms regenerative systems. Experiments in the subsonic regime have been carried out by Langener et al. [14]. The results show that the influence of the geometry and porosity of the probe of the porous material are small compared to the properties of the coolant, especially the specific heat. The composite carbon/carbon $(\mathrm{C} / \mathrm{C})$ materials used in these studies have been described by Selzer et al. [17]. A comprehensive summary of early and recent experimental investigations and system analysis on transpiration cooling can be found in the work of Langener [7].

Numerical investigations of transpiration cooling, based on fully simulating porous media and hot gas flow as well as temperature distribution, are still rare. Glass et al. [19] have presented a numerical investigation of transpiration cooling with $\mathrm{C} / \mathrm{C}$ materials at scramjet combustor conditions using hydrogen as a coolant. In a first step, they estimate the reduction of the heat flux at the wall due to the blocking provided by the injection using a boundary layer code. The flow through the porous material is then simulated with the aid of a one-dimensional finite difference code for the temperatures of solid and coolant and the pressure of the coolant. To our knowledge coupled computations have not been performed yet. 
In [20] Tully investigates transpiration cooling of an injector plate of liquid propellant rocket engines using a monolithic approach. Numerical simulations of both porous media and open channel flow are based on a semi heuristic model for the porous media flow which reduces to the NavierStokes equations for incompressible flow in the case of an open channel flow. Not quite in agreement with the targeted application, only air has been used as injected coolant and propellant.

Numerical simulations of transpiration cooling of a porous cylinder in a subsonic turbulent flow can be found in Mathelin et al. [21]. There a two-dimensional RANS simulation using a Reynolds Stress Model of the hot gas flow is coupled with a one-dimensional porous media solver where the flow rate is prescribed and only two temperature equations are solved in the porous medium. The injection of the coolant is modeled by source terms for mass, momentum and energy at the wall. Their results are compared with experimental results. As a conclusion thermal non-equilibrium between the coolant and the porous material is far from being negligible at least for lower blowing ratios.

A quite similar approach has been proposed by Langener, Cheuret et al. [22] [23] within the framework of the project Aerodynamic and Thermal Load Interactions with Lightweight Advanced Materials for High Speed Flight (ATLLAS) [24]. In this project, transpiration cooling has been investigated for Ramjet and Scramjet engines using a supersonic hot gas channel with a porous material integrated in the channel wall. In the same study a second numerical approach, similar to Tully [20], is described, using transport equations for a turbulent flow adapted to a porous media flow by introducing the porosity to the equations. Again this approach is monolithic with different coefficients in the two respective regimes. In contrast to the first numerical code, the second one does not account for thermal non-equilibrium in the porous material. The results of these two approaches are compared with experimental results and found to be in a reasonable agreement concerning the cooling efficiency.

Gascoin et al. [25] [26] employ an approach similar to the latter one to investigate the use of hydrocarbon fuel as a coolant for transpiration cooling, including the effects of chemical reactions due to fuel pyrolysis. Their numerical results are validated by experiments.

Hannemann [27] presents a numerical approach for simulating the injection of different coolants into a laminar, hypersonic boundary layer. A simplified one-dimensional model is used which assumes a given mass flux distribution and the temperature of the coolant being equal to the temperature of the surface of the porous wall. Results for different coolants are shown and the influence of the injection on the flow field is described.

However, in summary, it is fair to say that one still faces a severe lack of understanding concerning the fundamentals of the flow field and the thermal interaction in both the porous medium and near to the injection in the hot gas flow.

\subsection{Objective}

As mentioned before, further detailed basic research is necessary to guarantee the functionality and reliability of such a new cooling technique. To be able to go beyond the approaches mentioned above the present work puts forward coupled numerical simulations of porous media and pure fluid flow. In particular, in order to be able to adequately capture downstream effects near the interface between hot gas flow and porous medium flow, we propose in contrast to most previous studies a two dimensional model for the porous medium flow whose physical justification is given below in the 
next section. Specifically, a suitable mathematical model for pressure-driven porous medium flow is designed that consists of the continuity equation, the Darcy-Forchheimer equation and temperature equations for both solid and fluid. For convenience we have chosen a finite element solver using the deal.ii library [8] to approximate the porous medium flow. Physically reasonable coupling conditions are designed for the interface between the pure fluid flow and the porous material. The coupling is realized in a weak sense by successive alternating solves in the hot gas and porous medium regime using the results of the respective preceding calculation in one regime as a boundary condition for the other regime. Such a coupling can be tightened by iterating the computations for the same time level which in the limit corresponds to solving the coupled problem.

\subsection{Layout}

This paper is structured as follows: In Section 2 we develop a d-dimensional model for pressuredriven porous medium flow. Here the spatial dimension $d$ is either two or three. The numerical discretization of this model is described in Section 3. Subsequently, the coupling with a finite volume flow solver is outlined in Section 5. In Section 6 numerical simulations of transpiration cooling through a porous material are compared with experimental results. Finally, a conclusion is given in Section 7.

\section{THE MODEL}

In this section, the governing equations used for modeling the porous medium flow along with suitable initial and boundary conditions are derived.

\subsection{Governing equations}

In order to simulate the cooling gas injection into a hot gas flow through a porous material a pressuredriven flow in a porous medium is modeled by taking the compressibility of the fluid, the velocity of the fluid and the heat conduction in the fluid as well as the solid into account. The continuum model thus consists of the continuity equation, the Darcy-Forchheimer equation and two heat equations. We are particularly interested in properly capturing transport as well as non-equilibrium temperature effects. In the following the model is briefly summarized. A more detailed discussion can be found in [29] and references cited therein.

In contrast to pure fluids the porosity $\varphi$ of the porous medium has to be accounted for in the continuum model. The porosity of a porous material is defined as the ratio of void space and the total volume of the medium. Here we assume that all the void space is connected. Averaging the fluid velocity over a volume $V_{f}$ consisting only of fluid, the intrinsic average velocity $\mathbf{v}$ is obtained. This is related to the Darcy velocity (seepage velocity, filtration velocity, superficial velocity) $\mathbf{V}$, i.e., the average velocity with respect to a volume element $V_{m}$ comprizing both solid and fluid material, by the porosity as $\mathbf{V}=\varphi \mathbf{v}$. The continuity equation for the fluid density $\rho$ then reads

$$
\varphi \frac{\partial \rho}{\partial t}+\nabla \cdot(\rho \mathbf{V})=0
$$


For the momentum balance quadratic drag is included, resulting in the Darcy-Forchheimer equation

$$
\rho\left(\frac{1}{\varphi} \frac{\partial \mathbf{V}}{\partial t}+\frac{1}{\varphi^{2}}(\mathbf{V} \cdot \nabla) \mathbf{V}\right)=-\nabla p-\mu \mathbf{K}_{\mathbf{D}}^{-1} \mathbf{V}-\rho \mathbf{K}_{\mathbf{F}}^{-1}|\mathbf{V}| \mathbf{V}
$$

Here $\mu$ denotes the dynamic viscosity of the fluid, $\mathbf{K}_{\mathbf{D}}$ the permeability tensor of the medium and $\mathbf{K}_{\mathbf{F}}$ the Forchheimer coefficient, which is also a tensor. In the simulations presented in this paper, the contributions of the nonlinear Forchheimer term are small. The pressure $p$ is determined by the ideal gas law

$$
p=\rho T_{f} R
$$

with the specific gas constant $R$ and the fluid temperature $T_{f}$.

The temperature $T_{s}$ of the solid and $T_{f}$ of the fluid are assumed to be in non-equilibrium. Therefore two heat equations for both the solid and the fluid, respectively, are necessary. Since no convection takes place in the solid we obtain

$$
(1-\varphi) \rho_{s} c_{s} \frac{\partial T_{s}}{\partial t}=(1-\varphi) \nabla \cdot\left(\boldsymbol{\kappa}_{s} \nabla T_{s}\right)+h\left(T_{f}-T_{s}\right),
$$

where $\kappa_{s}$ is the heat conduction tensor, $\rho_{s}$ the constant density and $c_{s}$ the specific heat capacity of the solid. Since the fluid is in contact with the solid the exchange of heat is accounted for by the heat transfer coefficient $h$ to be determined by experiments.

Since the fluid moves convection occurs in the heat equation for the fluid

$$
\varphi \rho c_{p, f}\left(\frac{\partial T_{f}}{\partial t}+\frac{1}{\varphi} \mathbf{V} \cdot \nabla T_{f}\right)=\varphi \nabla \cdot\left(\kappa_{f} \nabla T_{f}\right)+h\left(T_{s}-T_{f}\right) .
$$

Here $\kappa_{f}$ is the heat conduction coefficient, which is equal in all directions and therefore a scalar and not a tensor, and $c_{p, f}$ is the specific heat capacity of the fluid at constant pressure.

\subsection{Initial and boundary conditions}

To obtain a well-posed problem, the differential equations have to be complemented by suitable boundary and initial conditions.

For the boundary $\Gamma$ of the domain occupied by the porous material we have to distinguish the inflow boundary $\Gamma_{R}$, where the cooling gas enters from the reservoir, and the outflow boundary $\Gamma_{H G}$ of the porous medium where the coolant leaves into the hot gas flow. In addition we have to consider the solid walls $\Gamma_{W}$ separating the porous medium from non-porous structure, see Figure 1.

Pressure and temperatures at the inflow boundary are determined by the respective reservoir conditions that are assumed to be constant and homogeneous. More precisely, denoting by $T_{b}$ the temperature of the solid on the backside of the porous material and the reservoir temperature, respectively, while $p_{c}, T_{c}$ stand for reservoir pressure and temperature of the coolant, the boundary conditions at $\Gamma_{R}$ read

$$
p(t, \mathbf{x})=p_{c}, \quad T_{s}(t, \mathbf{x})=T_{b}, \quad T_{f}(t, \mathbf{x})=T_{c} \quad \text { on } \quad \Gamma_{R} .
$$




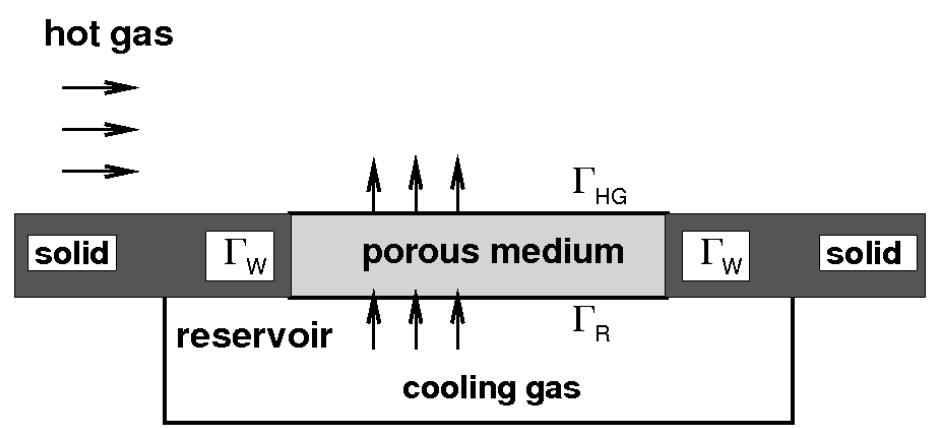

Figure 1. Configuration of coupled fluid-porous medium problem.

Recall that the fluid density $\rho$ can be computed by the ideal gas law (3) from these values which, in view of the ideal gas law (3), yields the boundary condition

$$
\rho(x, t)=\rho_{R}:=\frac{p_{c}}{T_{f} R} .
$$

Concerning the outflow boundary $\Gamma_{H G}$, the influence of the hot gas flow is modeled by the following boundary conditions

$$
p(t, \mathbf{x})=p_{H G}(t, \mathbf{x}), \quad-\boldsymbol{\kappa}_{s} \frac{\partial T_{s}}{\partial \mathbf{n}}(t, \mathbf{x})=h_{H G}\left(T_{s}(t, \mathbf{x})-T_{H G}(t, \mathbf{x})\right) \quad \text { on } \quad \Gamma_{H G},
$$

with the hot gas pressure $p_{H G}(t, \mathbf{x})$ and temperature $T_{H G}(t, \mathbf{x})$, which may vary in time and space. The heat transfer coefficient $h_{H G}$ describes the heat exchange between the solid part of the porous material and the hot gas flow. It should not be mistaken with the heat transfer coefficient $h$ between the solid and the fluid of the porous material. It strongly depends on the injected coolant mass flux. Therefore, $h_{H G}$ is modeled following the approach by Kays et al. [15].

The velocity component normal to the outflow boundary can then be computed from the mass flux of the coolant $\dot{m}$ measured in the experiments

$$
V_{y}=\frac{\dot{m}}{\rho_{H G} A_{P M}} \text { resp. } \quad \mathbf{V}_{H G}:=V_{y} \mathbf{e}_{\mathbf{d}} \quad \text { on } \boldsymbol{\Gamma}_{\mathbf{H G}}
$$

where $A_{P M}$ denotes the cross-section of the porous material and $\mathbf{e}_{\mathbf{d}}$ is the Cartesian unit vector in the outward normal direction to $\Gamma_{H G}$.

The bordering side-walls corresponding to $\Gamma_{W}$ are assumed to be adiabatic, i.e.,

$$
\frac{\partial T_{s}}{\partial \mathbf{n}}=0, \quad \frac{\partial T_{f}}{\partial \mathbf{n}}=0 \quad \text { on } \quad \Gamma_{W} .
$$

It remains to discuss $\mathbf{V}$ and $\rho$ on $\Gamma_{W}$ and on $\Gamma_{R}$. Of course, from a physical perspective, the component of $\mathbf{V}$ which is orthogonal to $\Gamma_{W}$ must vanish on $\Gamma_{W}$, i.e.,

$$
\mathbf{n} \cdot \mathbf{V}=0 \text { on } \Gamma_{W}
$$


while nontrivial tangential components have to be permitted, i.e., $\Gamma_{W}$ should be a characteristic boundary for transport system (1), (2). Since we have deliberately chosen an inviscid model to avoid boundary layers in the pores and at adiabatic walls we can prescribe boundary values only on inflow boundaries, namely on those parts of $\Gamma$ where the angle between the outward normal and the direction of $\mathbf{V}$ is larger than $90^{\circ}$. Thus, the type of $\Gamma_{W}$ depends on the velocity field itself and the nonlinearity allows $\mathbf{V}$ to adapt to a physically correct tangential field at $\Gamma_{W}$. Rather than enforcing tangentiality through a boundary condition, which would be "illegal" for the equation at hand, it will be a consequence of our linearization of the nonlinear system discussed later. Vanishing normal components of $\mathbf{V}$ at $\Gamma_{W}$ imply that $\Gamma_{W}$ is also a charcateristic boundary for the continutity equation so that no conditions for the density $\rho$ are needed.

Finally, suitable values for the initial conditions

$$
\rho(t=0)=\rho_{0}, \quad \mathbf{V}(t=0)=\mathbf{V}_{0}, \quad T_{s}(t=0)=T_{s, 0}, \quad T_{f}(t=0)=T_{f, 0}
$$

need to be chosen as detailed in Section 3.3.

\section{NUMERICAL METHOD}

Due to the form of the inflow boundary conditions and the dissipative nature of the heat equations as well as low velocities, the porous medium flow is not expected to develop discontinuities. Since we are at this point not primarily interested in the most efficient way of solving the equations (1) (4) we derive next a weak formulation that allows us to employ the deal.II library. Details on this library can be found in Bangerth et al. [8]. A potential domination of convective terms in (5) and the asymmetry of (2) may require in addition to stabilize the numerical discretization. For that purpose a standard choice would be an SUPG-stabilization (see e.g. [35]) adding viscosity only in streamwise direction. We shall refer to this finite element solver as the porous medium solver.

\subsection{Weak formulation}

The weak formulation of the equations (1) - (5) requires selecting appropriate function spaces. In view of the asymmetry of the first order components in these equations, it is natural to choose the trial spaces different from the test spaces as argued next.

Multiplying the continuity equation (1) by the test function $\tilde{\rho}$ and integrating over the domain $\Omega$, yields

$$
\int_{\Omega} \tilde{\rho} \nabla \cdot(\rho \mathbf{V})=0
$$

which should hold for all $\tilde{\rho} \in Y_{\rho}:=L_{2}(\Omega)$ where $L_{2}(\Omega)$ denotes the space of all square integrable functions. For the left-hand side to be well-defined we need $\rho \mathbf{V} \in H^{1}(\Omega)^{d}$ where $H^{1}(\Omega)$ denotes the standard Sobolev space comprised of all square integrable functions whose first order weak derivatives are also square integrable.

We postpone specifying suitable trial spaces for $\rho, \mathbf{V}$ after discussing next the momentum equations. 
Multiplying the Darcy-Forchheimer equation (2) from the left by test functions $\tilde{\mathbf{V}}$ and substituting the pressure with the aid of the ideal gas law (3), yields

$$
\begin{aligned}
\frac{1}{\varphi^{2}} \int_{\Omega} \tilde{\mathbf{V}} \cdot \rho(\mathbf{V} \cdot \nabla) \mathbf{V}= & -\int_{\Omega} \tilde{\mathbf{V}} \cdot \nabla p-\mu \int_{\Omega} \tilde{\mathbf{V}} \cdot \mathbf{K}_{\mathbf{D}}^{-1} \mathbf{V}-\int_{\Omega} \tilde{\mathbf{V}} \cdot \mathbf{K}_{\mathbf{F}}^{-1} \rho|\mathbf{V}| \mathbf{V} \\
= & -\int_{\Omega} \tilde{\mathbf{V}} \cdot \nabla\left(\rho R T_{f}\right)-\mu \int_{\Omega} \tilde{\mathbf{V}} \cdot \mathbf{K}_{\mathbf{D}}^{-1} \mathbf{V} \\
& -\int_{\Omega} \tilde{\mathbf{V}} \cdot \mathbf{K}_{\mathbf{F}}^{-1} \rho|\mathbf{V}| \mathbf{V}
\end{aligned}
$$

which is again to hold for all $\tilde{\mathbf{V}}$ from a suitable test class $Y_{v}$. As we shall see any space $Y_{v}$ which is dense in $L_{2}(\Omega)^{d}$ suffices. In fact, taking in view of (7) and (9),

$$
\begin{aligned}
& \rho \in X_{\rho} \quad:=\left\{\rho \in H^{1}(\Omega) \cap L_{\infty}(\Omega):\left.\rho\right|_{\Gamma_{R}}=\rho_{R}\right\}, \\
& \mathbf{V} \in X_{v} \quad:=\left\{\mathbf{V} \in H^{1}(\Omega)^{d} \cap L_{\infty}(\Omega)^{d}:\left.\mathbf{V}\right|_{\Gamma_{H G}}=\mathbf{V}_{H G}\right\},
\end{aligned}
$$

all volume integrals in (13), (14) are indeed well-defined when

$$
\tilde{\rho} \in Y_{\rho}:=H_{0, \Gamma_{R}}^{1}(\Omega), \quad \tilde{\mathbf{V}} \in Y_{v}:=\left(H_{0, \Gamma_{H G}}^{1}(\Omega)\right)^{d} .
$$

This is clear for the left-hand side of (13). Since $\rho \in L_{\infty}(\Omega), \mathbf{V} \in L_{2}(\Omega)^{d}$ we have $\rho(\mathbf{V} \cdot \nabla) \mathbf{V} \in$ $L_{2}(\Omega)^{d}$ which takes care of the left-hand side of (14). The right-hand side of (14) can be treated in a similar fashion, noting that $T_{f}$ as a solution to an elliptic problem belongs to $H^{1}(\Omega)$.

Finally, both elliptic temperature equations (4), (5), are treated along standard lines. After multiplying by suitable test functions $\tilde{T}_{s}$ the conduction terms are integrated by parts and Gauss' theorem is applied. As for (4), this provides

$$
\begin{aligned}
0 & =(1-\varphi) \int_{\Omega} \tilde{T}_{s} \nabla \cdot\left(\boldsymbol{\kappa}_{\boldsymbol{s}} \nabla T_{s}\right)+h \int_{\Omega} \tilde{T}_{s}\left(T_{f}-T_{s}\right) \\
& =-(1-\varphi) \int_{\Omega} \nabla \tilde{T}_{s} \cdot \boldsymbol{\kappa}_{\boldsymbol{s}} \nabla T_{s}+(1-\varphi) \int_{\Gamma} \tilde{T}_{s} \boldsymbol{\kappa}_{\boldsymbol{s}} \frac{\partial T_{s}}{\partial \mathbf{n}}+h \int_{\Omega} \tilde{T}_{s}\left(T_{f}-T_{s}\right) .
\end{aligned}
$$

Now notice that Dirichlet boundary conditions for $T_{s}$ are only imposed on $\Gamma_{R}$, see (6). Therefore, the affine space

$$
X_{T_{s}}:=\left\{T \in H^{1}(\Omega):\left.T\right|_{\Gamma_{R}}=T_{b}\right\},
$$

Hence, the test functions $\tilde{T}_{s}$ have to belong to $Y_{T_{s}}:=H_{0, \Gamma_{R}}^{1}(\Omega)$ which is the subspace of those elements in $H^{1}(\Omega)$ whose trace vanishes on $\Gamma_{R}$. Inserting now the Neumann boundary conditions (10) on $\Gamma_{W}$ and the Robin-type boundary conditions (8), yields

$$
\begin{aligned}
0= & -(1-\varphi) \int_{\Omega} \nabla \tilde{T}_{s} \cdot \kappa_{s} \nabla T_{s}-(1-\varphi) h_{H G} \int_{\Gamma_{H G}} \tilde{T}_{s}\left(T_{s}-T_{H G}\right) \\
& +h \int_{\Omega} \tilde{T}_{s}\left(T_{f}-T_{s}\right), \quad \tilde{T}_{s} \in H_{0, \Gamma_{R}}^{1}(\Omega)=: Y_{T_{s}} .
\end{aligned}
$$


Proceeding similarly with (5), taking the Dirichlet boundary condition (6) for $T_{f}$ on $\Gamma_{R}$ into account, a suitable trial space is again the affine space

$$
X_{T_{f}}:=\left\{T \in H^{1}(\Omega):\left.T\right|_{\Gamma_{R}}=T_{c}\right\},
$$

so that the corresponding test space is again $Y_{T_{f}}:=H_{0, \Gamma_{R}}^{1}(\Omega)$ from which the test functions $\tilde{T}_{f}$ should be taken to obtain

$$
\begin{aligned}
\int_{\Omega} \tilde{T}_{f} \rho c_{p, f} \mathbf{V} \cdot \nabla T_{f}= & \varphi \int_{\Omega} \tilde{T}_{f} \nabla \cdot\left(k_{f} \nabla T_{f}\right)+h \int_{\Omega} \tilde{T}_{f}\left(T_{s}-T_{f}\right) \\
= & -\varphi k_{f} \int_{\Omega} \nabla \tilde{T}_{f} \cdot \nabla T_{f}+\varphi k_{f} \int_{\Gamma_{H G}} \tilde{T}_{f} \frac{\partial T_{f}}{\partial \mathbf{n}} \\
& +h \int_{\Omega} \tilde{T}_{f}\left(T_{s}-T_{f}\right), \quad \tilde{T}_{f} \in H_{0, \Gamma_{R}}^{1}(\Omega):=Y_{T_{f}} .
\end{aligned}
$$

Note that the quantity $\frac{\partial T_{f}}{\partial \mathbf{n}}$ is not yet determined and will be taken from information provided by the hot gas flow.

In summary, the weak formulation of the equations (1) - (5) requires finding $\mathbf{U}_{p m}=$ $\left(\rho, \mathbf{V}, T_{s}, T_{f}\right) \in X:=X_{\rho} \times X_{v} \times X_{T_{s}} \times X_{T_{f}}$ such that for all $\Theta_{p m}=\left(\tilde{\rho}, \tilde{\mathbf{V}}, \tilde{T}_{s}, \tilde{T}_{f}\right) \in Y:=Y_{\rho} \times$ $Y_{v} \times Y_{T_{s}} \times Y_{T_{f}}$ one has

$$
a^{p m}\left(\mathbf{U}_{p m}, \Theta_{p m}\right)=F\left(\Theta_{p m}\right), \quad \Theta_{p m} \in Y,
$$

where

$$
F\left(\Theta_{p m}\right)=(1-\varphi) h_{H G} \int_{\Gamma_{H G}} \tilde{T}_{s} T_{H G}
$$

and

$$
a^{p m}\left(\mathbf{U}_{p m}, \Theta_{p m}\right)=a_{\rho}\left(\mathbf{U}_{p m}, \Theta_{p m}\right)+a_{v}\left(\mathbf{U}_{p m}, \Theta_{p m}\right)+a_{T_{s}}\left(\mathbf{U}_{p m}, \Theta_{p m}\right)+a_{T_{f}}\left(\mathbf{U}_{p m}, \Theta_{p m}\right)
$$

with

$$
\begin{aligned}
a_{\rho}\left(\mathbf{U}_{p m}, \Theta_{p m}\right) & :=\int_{\Omega} \tilde{\rho} \nabla \cdot(\rho \mathbf{V}) \\
a_{v}\left(\mathbf{U}_{p m}, \Theta_{p m}\right) & :=-\varphi^{-2} \int_{\Omega} \tilde{\mathbf{V}} \cdot\left(\nabla\left(\rho R T_{f}\right)+\mu \mathbf{K}_{\mathbf{D}}^{-1} \mathbf{V}+\mathbf{K}_{\mathbf{F}}^{-1} \rho|\mathbf{V}| \mathbf{V}\right)
\end{aligned}
$$

and

$$
\begin{aligned}
& a_{T_{s}}\left(\mathbf{U}_{p m}, \Theta_{p m}\right):=(1-\varphi)\left\{\int_{\Omega} \nabla \tilde{T}_{s} \cdot \boldsymbol{\kappa}_{s} \nabla T_{s}+h_{H G} \int_{\Gamma_{H G}} \tilde{T}_{s} T_{s}\right\}-h \int_{\Omega} \tilde{T}_{s}\left(T_{f}-T_{s}\right) \\
& a_{T_{f}}\left(\mathbf{U}_{p m}, \Theta_{p m}\right):=\int_{\Omega} \tilde{T}_{f}\left(\rho c_{p, f} \mathbf{V} \cdot \nabla T_{f}+h\left(T_{f}-T_{s}\right)\right)+\varphi k_{f} \nabla \tilde{T}_{f} \cdot \nabla T_{f}-\int_{\Gamma_{H G}} \varphi k_{f} \tilde{T}_{f} \frac{\partial T_{f}}{\partial \mathbf{n}} .
\end{aligned}
$$


To be later able to formulate proper coupling conditions between the hot gas and porous medium flow we shall write

$$
F\left(\Theta_{p m}\right)=F\left(\Theta_{p m} ; T_{H G}\right),
$$

to express the dependence of the right-hand side functional $F$ in (22) on data that will eventually come from the hot gas flow in the channel.

\subsection{Discretization}

To approximate the solution of (22) we choose a suitable finite element trial space $X_{h} \subset X$ and a corresponding test space $Y_{h} \subset Y$, both being Cartesian products of finite element spaces for the individual components of $X, Y$, respectively, incorporating the respective boundary constraints given in (15), (16), (18), (20). The Dirichlet conditions (6) on $\Gamma_{R}$ appearing in the elliptic part (19), (21) are incorporated as essential boundary conditions in the corresponding trial spaces which are therefore affine and not linear. All boundary conditions in the hyperbolic part are natural ones enforced weakly through the right-hand side functional.

Since all component spaces are subspaces of $H^{1}$, we start of using multilinear finite elements for all spaces. One then has to solve

$$
a^{p m}\left(\mathbf{U}_{p m}^{h}, \Theta_{p m}^{h}\right)=F\left(\Theta_{p m}^{h}\right), \quad \Theta_{p m}^{h} \in Y_{h} .
$$

More specifically, denoting by $\left\{\mathbf{U}_{p m}^{i}: i=1, \ldots, N_{h}\right\},\left\{\Theta_{p m}^{i}: i=1, \ldots, N_{h}\right\}$ bases for $X_{h}, Y_{h}$, respectively, (30) amounts to finding for each of the physical quantities $\zeta \in\left\{\rho, V_{1}, \ldots, V_{d}, T_{f}, T_{s}\right\}$ the array $\left(u_{i}^{\zeta}: i=1, \ldots, N_{h}\right)$ for which $\left(\mathbf{U}_{p m}^{h}\right)_{\zeta}=\sum_{i=1}^{N_{h}} u_{i}^{\zeta}\left(\mathbf{U}_{p m}^{i}\right)_{\zeta}$ satisfies

$$
a^{p m}\left(\mathbf{U}_{p m}^{h}, \Theta_{p m}^{i}\right)=F\left(\Theta_{p m}^{i}\right), \quad i=1, \ldots, N_{h},
$$

which is a nonlinear system of equations of size $N_{h}$.

\subsection{Solving the Discrete Problem}

Since the first two components of (30) corresponding to (13) and (14) form a nonlinear hyperbolic system while the last two components corresponding to (19), and (21) is a linear parabolic system, we employ operator splitting to solve (31). More specifically, splitting the unknown $\mathbf{U}_{p m}^{h}$ into the convective and diffusive components $\mathbf{U}_{p m}^{h}=\left[\mathbf{U}_{t r}^{h}, \mathbf{T}^{h}\right]$ with $\mathbf{U}_{t r}^{h}=\left(\rho_{h}, \mathbf{V}_{h}\right), \mathbf{T}^{h}=\left(T_{s, h}, T_{f, h}\right)$ we alternate solving the parabolic system

$$
\begin{aligned}
& a_{T_{s}}\left(\left[\mathbf{U}_{t r}^{h, \text { old }}, \mathbf{T}^{h, \text { new }}\right], \Theta_{p m}^{h}\right)+a_{T_{f}}\left(\left[\mathbf{U}_{t r}^{h, \text { old }}, \mathbf{T}^{h, \text { new }}\right], \Theta_{p m}^{h}\right)= \\
& F\left(\Theta_{p m}^{h}\right)+a_{\rho}\left(\mathbf{U}_{p m}^{h, o l d}, \Theta_{p m}^{h}\right)+a_{v}\left(\mathbf{U}_{p m}^{h, \text { old }}, \Theta_{p m}^{h}\right), \quad \Theta_{p m}^{h} \in Y_{h},
\end{aligned}
$$

and the hyperbolic system

$$
\begin{aligned}
& a_{\rho}\left(\left[\mathbf{U}_{t r}^{h, \text { new }}, \mathbf{T}^{h, \text { old }}\right], \Theta_{p m}^{h}\right)+a_{v}\left(\left[\mathbf{U}_{t r}^{h, \text { new }}, \mathbf{T}^{h, \text { old }}\right], \Theta_{p m}^{h}\right)= \\
& F\left(\Theta_{p m}^{h}\right)+a_{T_{s}}\left(\mathbf{U}_{p m}^{h, o l d}, \Theta_{p m}^{h}\right)+a_{T_{f}}\left(\mathbf{U}_{p m}^{h, o l d}, \Theta_{p m}^{h}\right), \quad \Theta_{p m}^{h} \in Y_{h},
\end{aligned}
$$


where the forms $a_{\rho}, a_{v}, a_{T_{s}}, a_{T_{f}}$ are given by (25) - (28). More precisely, in order to account for the hyperbolic character of (33), one should employ streamline-upwind Petrov-Galerkin (SUPG) concepts which roughly means that the test functions $\Theta_{p m}^{h}$ are replaced in (33) and (32) by the quantities $\Theta_{p m}^{h}+\delta \mathbf{V} \cdot \Theta_{p m}^{h}$, see [30] [31]. More precisely, the terms $\delta \mathbf{V} \cdot \Theta_{p m}^{h}$ are added elementwise, due to the lack of regularity in the second order terms. The parameter $\delta$ should be chosen judiciously, see the discussion of numerical results later. Since $|\mathbf{V}|$ does not strongly dominate the diffusion coefficients, this stabilization can actually be omitted in (32).

The iteration is initiated by solving (32) first which we refer to as the outer iteration in the porous medium solver. Since the two temperature equations are linear, the linear system of equations evolving from the finite element discretization can be solved using any solver for non symmetric linear systems of equations. Depending on the size of the discretization, mainly depending on the number of space dimensions, either a sparse direct solver (UMFPack [32] [33]), which employs a direct LU factorization, or the BiCGstab method is used. The hyperbolic part (33) is nonlinear and has to be solved iteratively as well, which we call the inner iteration. Each corresponding linear problem is again solved with either the direct solver or the BiCGstab method. Here we simply use a Picard iteration, freezing alternatingly $\mathbf{V}_{h}, \rho_{h}$ from the preceding step. In particular, this allows us to conveniently ensure the slip boundary conditions at $\Gamma_{W}$, see (11), which do therefore not have to be incorporated into the trial space $X_{v}$, see (15).

The initiating solve of (32) as well the iterative solution of (33) require an initial guess for $\rho$ and $\mathbf{V}$. The initial density $\rho^{0}$ is determined by linearly interpolating the reservoir density $\rho_{f, R}$ and the hot gas density $\rho_{H G}$ in the $d$ th coordinate direction which is orthogonal to $\Gamma_{H G}$ and $\Gamma_{R}$. The densites $\rho_{f, R}$ and $\rho_{H G}$ are computed from the respective temperatures $T_{f, R}$ and $T_{H G}$ and pressures $p_{c}$ and $p_{H G}$ using the ideal gas law (3). The initial velocity field in $\Omega$ is then computed from the mass flux measured in the experiment and the initial density distribution

$$
\mathbf{V}^{\mathbf{0}}:=\frac{\dot{m}}{\rho^{0} A_{P M}} \mathbf{e}_{\mathbf{d}}
$$

where $\mathbf{e}_{\mathbf{d}}$ is the Cartesian unit vector in the inward normal direction to $\Gamma_{R}$.

\section{FLOW SOLVER}

In this section we briefly describe the flow solver for the pure fluid and corresponding discretizations that will be used in the actual computations.

\subsection{Flow Model}

We shall consider non-stationary flows since this conveniently accommodates classical turbulence models. Specifically, turbulent flows can be described by the Reynolds-averaged Navier-Stokes equations (RANS), which are obtained by applying the Reynolds-averaging

$$
f(\mathbf{x}, t)=\bar{f}(\mathbf{x}, t)+f^{\prime}(\mathbf{x}, t) \quad \text { with } \quad \bar{f}(\mathbf{x}, t):=\lim _{\Delta \rightarrow \infty} \frac{1}{\Delta} \int_{t}^{t+\Delta} f(\mathbf{x}, \tau) d \tau
$$


to the compressible Navier-Stokes equations. In contrast to incompressible flows, the resulting equations have a rather complex form due to fluctuations in the density. To simplify the representation, we employ in addition mass-averaging also referred to as the Favre-averaging

$$
f=\tilde{f}+f^{\prime \prime} \quad \text { with } \quad \tilde{f}:=\frac{\overline{\rho f}}{\bar{\rho}} .
$$

The resulting Favre-averaged Navier-Stokes equations in non-dimensional form using the Einstein summation convention read

$$
\begin{aligned}
\frac{\partial \bar{\rho}}{\partial t}+\frac{\partial\left(\bar{\rho} \widetilde{v}_{j}\right)}{\partial x_{j}} & =0 \\
\frac{\partial\left(\bar{\rho} \widetilde{v}_{i}\right)}{\partial t}+\frac{\partial\left(\bar{\rho} \widetilde{v}_{i} \widetilde{v}_{j}\right)}{\partial x_{j}} & =-\frac{\partial \bar{p}}{\partial x_{i}}+\frac{1}{R e_{r e f}} \frac{\partial \tau_{i j}^{e f f}}{\partial x_{j}} \\
\frac{\partial(\bar{\rho} \widetilde{E})}{\partial t}+\frac{\partial \widetilde{v}_{j}(\bar{\rho} \widetilde{E}+\bar{p})}{\partial x_{j}} & =\frac{1}{R e_{r e f}}\left(\frac{\partial}{\partial x_{j}}\left(\widetilde{v}_{i} \tau_{i j}^{e f f}-q_{j}^{e f f}\right)+\frac{\partial}{\partial x_{j}}\left(\overline{v_{i}^{\prime \prime} \tau_{i j}}-\frac{\overline{\rho v_{i}^{\prime \prime} v_{i}^{\prime \prime} v_{j}^{\prime \prime}}}{2}\right)\right),
\end{aligned}
$$

where $\rho$ denotes the density, $p$ the static pressure, $v_{i}$ the components of the fluid velocity $\mathbf{v}$ and $E=e+1 / 2 \mathbf{v}^{2}$ the total energy. The effective stress tensor $\tau_{i j}^{e f f}$ and the effective heat flux vector $q_{j}^{e f f}$ are given by

$$
\begin{aligned}
\tau_{i j}^{e f f} & :=\bar{\tau}_{i j}-\bar{\rho} \widetilde{R}_{i j}=\mu\left(2 \widetilde{S}_{i j}-\frac{2}{3} \widetilde{S}_{k k} \delta_{i j}\right)-\overline{\rho v_{i}^{\prime \prime} v_{j}^{\prime \prime}} \\
q_{j}^{e f f} & :=\bar{q}_{j}+q_{j}^{t}=-k \frac{\overline{\partial T}}{\partial x_{j}}+c_{v} \overline{\rho T^{\prime \prime}} .
\end{aligned}
$$

Here $\widetilde{R}_{i j}$ is called the Reynolds stress tensor and $q_{j}^{t}$ is the turbulent heat flux. $S=\left(S_{i j}\right)$ with $S_{i j}=\frac{1}{2}\left(\frac{\partial v_{i}}{\partial x_{j}}+\frac{\partial v_{j}}{\partial x_{i}}\right)$ denotes the strain rate tensor, $c_{v}$ the heat capacity at constant volume and $T$ is the temperature. $\tau_{i j}$ and $q_{j}$ are the components of the stress tensor and the heat flux vector, modeled as an isentropic Newtonian fluid and by Fourier's law, respectively. Air is modeled as a perfect gas. The dynamic viscosity $\mu$ is described by Sutherland's law and $k$ is the heat flux coefficient.

In order to close the system determined by the Favre-averaged Navier-Stokes equations (37), (38), (39) and the effective quantities (40), (41), the terms $\overline{\rho v_{i}^{\prime \prime} v_{j}^{\prime \prime}}, \overline{\rho T^{\prime \prime}}, \overline{v_{i}^{\prime \prime} \tau_{i j}}, \overline{\rho v_{i}^{\prime \prime} v_{i}^{\prime \prime} v_{j}^{\prime \prime}}$ have to be modeled. The most important step for closing this set of equations is the modeling of the Reynolds stress tensor. For this purpose, the Menter shear stress transport (SST) model [6] is applied. This model combines the advantages of a $k-\omega$ model near the wall, in the inner boundary layer, with the less sensitive behavior of a $k-\epsilon$ model in free stream turbulence. For two-equation eddy-viscosity models, the Boussinesq hypothesis is used to relate the Reynolds stresses to the mean velocities of the flow, i.e., $\widetilde{R}_{i j}=\overline{\rho v_{i}^{\prime \prime} v_{j}^{\prime \prime}} / \bar{\rho} \approx-2 \mu^{t} \widetilde{S}_{i j}-\frac{2}{3} k \delta_{i j}$. The turbulence eddy viscosity $\mu^{t}$ is therefore computed from two turbulent variables, which are for the Menter SST model the turbulent kinetic energy $k$ and the specific dissipation rate $\omega$. For these two variables the additional transport 
solid wall

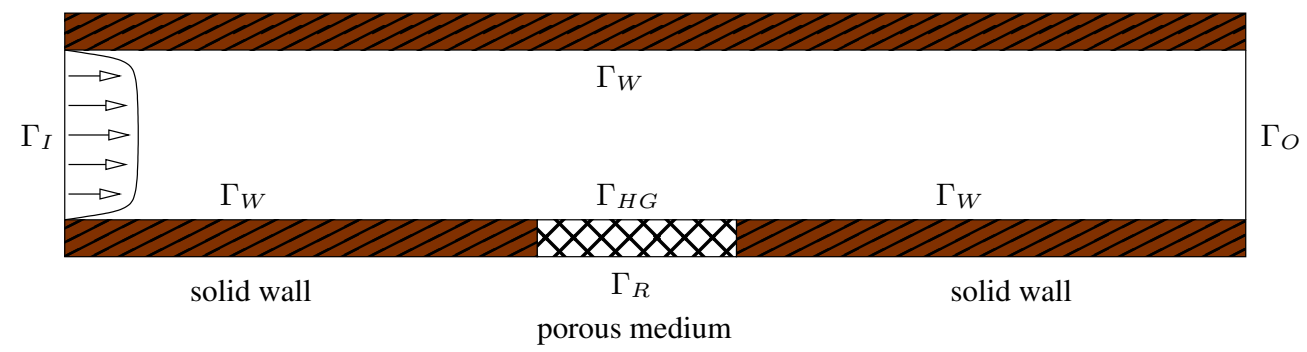

Figure 2. Computational domain of coupled fluid-porous medium problem in a channel.

equations

$$
\begin{aligned}
\frac{\partial \bar{\rho} k}{\partial t}+\frac{\bar{\rho} \widetilde{v}_{j} \partial k}{\partial x_{j}} & =P-\beta^{*} \bar{\rho} k \omega+\frac{\partial}{\partial x_{j}}\left(\left(\mu+\sigma_{k} \mu^{t}\right) \frac{\partial k}{\partial x_{j}}\right), \\
\frac{\partial \bar{\rho} \omega}{\partial t}+\frac{\partial \bar{\rho} \widetilde{v}_{j} \omega}{\partial x_{j}} & =\frac{\widetilde{\rho} \gamma}{\mu^{t}} P-\beta^{*} \bar{\rho} \omega^{2}+\frac{\partial}{\partial x_{j}}\left(\left(\mu+\sigma_{\omega} \mu^{t}\right) \frac{\partial \omega}{\partial x_{j}}\right)+2\left(1-F_{1}\right) \frac{\bar{\rho} \sigma_{\omega 2}}{\omega} \frac{\partial k}{\partial x_{j}} \frac{\partial \omega}{\partial x_{j}}
\end{aligned}
$$

have to be added to the system. Here the turbulence eddy viscosity $\mu^{t}$ is related to $k, \omega$ by $\mu^{t}=\frac{\alpha_{1} k}{\max \left\{\alpha_{1} \omega, \Omega F_{2}\right\}}$. In general, the production term $P=\widetilde{\tau}_{i j} \frac{\partial \widetilde{v}_{i}}{\partial x_{j}}$ is limited. For more details on the model and the involved parameters, such as $F_{1}=F_{1}(k, \omega), F_{2}=F_{2}(k, \omega), \alpha_{1}, \beta^{*}, \sigma_{k}, \sigma_{\omega}, \sigma_{\omega 2}$, $\Omega$, we refer to Menter [6].

The turbulent heat flux is modeled using a Fourier type approach $q_{j}^{t}=-\kappa^{t} \frac{\partial \widetilde{T}}{\partial x_{j}}$, where $\kappa^{t}=\frac{c_{p} \mu^{t}}{P r^{t}}$ is the eddy heat conductivity determined by the specific heat capacity $c_{p}$ at constant pressure and the turbulent Prandtl number $\operatorname{Pr}^{t}$ that is set to 0.9.

Finally, the diffusion of the turbulent kinetic energy associated with $\frac{\partial}{\partial x_{j}}\left(\overline{v_{i}^{\prime \prime} \tau_{i j}}-\frac{\overline{\rho v_{i}^{\prime \prime} v_{i}^{\prime \prime} v_{j}^{\prime \prime}}}{2}\right)$ is neglected for eddy viscosity models.

In summary, setting $\mathbf{U}_{N S}=(\bar{\rho}, \bar{\rho} \widetilde{\mathbf{v}}, \bar{\rho} \widetilde{E}, \bar{\rho} k, \bar{\rho} \omega)^{T}$ and

$$
\mathbf{L}_{N S}\left(\mathbf{U}_{N S}\right):=\left(\begin{array}{c}
\nabla \cdot(\bar{\rho} \widetilde{\mathbf{v}}) \\
\nabla \cdot\left(\bar{\rho} \widetilde{\mathbf{v}}^{T} \widetilde{\mathbf{v}}+\bar{p}\right)-\frac{1}{R e_{r e f}} \nabla \cdot \tau^{e f f} \\
\nabla \cdot(\widetilde{\mathbf{v}}(\bar{\rho} \widetilde{E}+\bar{p}))-\frac{1}{R e_{r e f}} \nabla \cdot\left(\tau^{e f f} \widetilde{\mathbf{v}}-\mathbf{q}^{e f f}\right) \\
\frac{\bar{\rho} \widetilde{v}_{j} \partial k}{\partial x_{j}}-P+\beta^{*} \bar{\rho} k \omega-\frac{\partial}{\partial x_{j}}\left(\left(\mu+\sigma_{k} \mu^{t}\right) \frac{\partial k}{\partial x_{j}}\right) \\
\frac{\partial \bar{\rho} \widetilde{v}_{j} \omega}{\partial x_{j}}-\frac{\gamma \widetilde{\rho}}{\mu^{t}} P+\beta^{*} \bar{\rho} \omega^{2}-\frac{\partial}{\partial x_{j}}\left(\left(\mu+\sigma_{\omega} \mu^{t}\right) \frac{\partial \omega}{\partial x_{j}}\right)-2\left(1-F_{1}\right) \frac{\bar{\rho} \sigma_{\omega 2}}{\omega} \frac{\partial k}{\partial x_{j}} \frac{\partial \omega}{\partial x_{j}}
\end{array}\right),
$$

the Favre-averaged Navier-Stokes equations (37)-(39) and the transport equations (42), (43) from the Menter SST model then read

$$
\frac{\partial \mathbf{U}_{N S}}{\partial t}+\mathbf{L}_{N S}\left(\mathbf{U}_{N S}\right)=\mathbf{0}
$$

These equations need to be complemented by suitable initial and boundary conditions detailed next. A sketch of the computational domain can be seen in Fig. 2. At the inflow boundary $\Gamma_{I}$ a turbulent profile for temperature and velocity is prescribed using the law of the wall. At the outflow boundary $\Gamma_{O}$ only the pressure is prescribed. The channel walls are adiabatic and no-slip boundary conditions 
are imposed. The boundary portion $\Gamma_{H G}$ deserves special attention. Instead of no-slip conditions we require

$$
\left.\mathbf{v}_{N S}\right|_{\Gamma_{H G}}=\left.\mathbf{V}^{*}\right|_{\Gamma_{H} G},\left.\quad T_{N S}\right|_{\Gamma_{H G}}=\left.T^{*}\right|_{\Gamma_{H G}},
$$

where $\mathbf{V}^{*}, T^{*}$ are given and determine the total energy on $\Gamma_{H G}$. Later these data will come from the porous medium flow and we briefly express these relations in what follows by

$$
c_{H G}\left(\mathbf{U}_{N S} ; \mathbf{V}^{*}, T^{*}\right)=0 .
$$

\subsection{Discretization}

The flow solver Quadflow [2] solves (45) using a cell-centered fully adaptive finite volume method on locally refined grids. Mesh adaptation is based on multiscale analysis [3] instead of classical gradient- or residual-based error estimators. The computational grids are represented by blockstructured parametric B-Spline patches [4] to deal with complex geometries. In order to reduce the computational load to an acceptable amount, these tools are equipped with parallelization techniques based on space-filling curves [5] to run the simulations on distributed memory architectures.

The convective fluxes are determined by solving quasi-one-dimensional Riemann problems at the cell interfaces. Several approximate Riemann solvers (Roe, HLLC, AUSMDV) and upwind schemes (van Leer) have been incorporated. A linear, multidimensional reconstruction of the conservative variables is applied to increase the spatial accuracy. In order to avoid oscillations in the vicinity of local extrema and discontinuities, limiters with TVD property are used. Concerning the computation of the viscous fluxes, the gradients of the variables at cell interfaces are determined using the divergence theorem. Finally, the time-integration is performed by an explicit multistage Runge-Kutta scheme and a fully implicit Newton-Krylov type method, respectively.

In our subsequent computations we are interested in stationary solutions. Therefore, the time variable $t$ will be used as a relaxation parameter. Since time accuracy plays no role we shall employ a simple backward Euler discretization with a specific strategy for increasing time increments in the course of the solution process. This will be described later in the applications in more detail. At this point it suffices to note that the discrete counterpart of (45) reads

$$
(\Delta t)^{-1} \mathbf{U}_{N S}^{n+1}+\mathbf{L}_{N S}^{h}\left(\mathbf{U}_{N S}^{n+1}\right)=(\Delta t)^{-1} \mathbf{U}_{N S}^{n}, \quad n=0,1,2, \ldots
$$

\section{COUPLING BOTH FLOW REGIMES}

We are ultimately interested in finding solutions $\mathbf{U}_{N S}, \mathbf{U}_{P M}$ in both flow regimes arising from the mutual interactions between both media. This interaction takes place at the boundary portion $\Gamma_{H G}$ and results in the following fully coupled system

$$
\begin{aligned}
\mathbf{L}_{N S}\left(\mathbf{U}_{N S}\right) & =0, & 0=c_{H G}\left(\mathbf{U}_{N S} ; \mathbf{V}_{P M},\left.T_{s}\right|_{\Gamma_{H G}}\right) ; \\
a^{p m}\left(\mathbf{U}_{P M}, \Theta\right) & =F\left(\Theta ; T_{H G}\right), & \forall \Theta \in Y .
\end{aligned}
$$

In the first system the structure temperature $T_{s}$ from the porous medium and the velocity field $\mathbf{V}_{P M}$ enter as data for the hot gas channel flow where, in analogy to (9), the velocity field $\mathbf{V}_{P M}$ 
is given by $\dot{m} /\left(\rho_{P M} A_{P M}\right) \mathbf{e}_{d}$ with $\rho_{P M}=\left.\rho\right|_{\Gamma_{H G}}$, the trace of the coolant density on $\Gamma_{H G}$. In the second system the temperature $T_{H G}$ determines the right-hand side functional in the porous medium system. Furthermore, $\mathbf{V}_{H G}$, given by (9), also provided by the hot gas flow $\mathbf{U}_{N S}$, enters as boundary conditions, see (15) .

Note that the cooling gas injection through the porous material is treated here as a fully laminar flow in all computations since the turbulent kinetic energy is set to zero. For $\omega$ the far field value

$$
\omega_{e}=\frac{\rho_{e} k_{e}}{\mu_{t, e}}
$$

is chosen, where the initial value for the turbulent viscosity is computed by the user defined ratio between the turbulent and the laminar viscosity

$$
\frac{\mu_{t, e}}{\mu_{l, e}}=0.001
$$

This is reasonable because $\omega$ does not produce any turbulence.

The stationary equilibrium solution of the coupled system is then determined by the underlying modeling assumption of the continuity of the pressure at the interface, i.e.,

$$
\left.p\left(\mathbf{U}_{N S}\right)\right|_{\Gamma_{H G}}=\left.p\left(\mathbf{U}_{P M}\right)\right|_{\Gamma_{H G}} .
$$

We solve (49) again by operator splitting. Starting with the porous medium (properly initializing the hot gas flow data at $\Gamma_{H G}$ as detailed later), we alternatingly solve the systems

$$
a^{p m}\left(\mathbf{U}_{P M}^{n+1}, \Theta\right)=F\left(\Theta ; T_{H G}^{n}\right), \quad \forall \Theta \in Y,
$$

and

$$
\mathbf{L}_{N S}\left(\mathbf{U}_{N S}^{n+1}\right)=0, \quad 0=c_{H G}\left(\mathbf{U}_{N S}^{n+1} ; \mathbf{V}_{P M}^{n},\left.T_{s}^{n}\right|_{\Gamma_{H G}}\right) ;
$$

approximately, employing the discretizations described before, until

$$
\left\|\left.p\left(\mathbf{U}_{N S}^{n}\right)\right|_{\Gamma_{H G}}-\left.p\left(\mathbf{U}_{P M}^{n}\right)\right|_{\Gamma_{H G}}\right\|_{L_{1}} / p_{\infty} \leq \varepsilon
$$

for a given tolerance $\varepsilon>0$. In our numerical experiments discussed later we use $\varepsilon=5 \times 10^{-4}$.

There are several options to deal with the discrete problems. The problem (53) has been discussed before. A favorable alternative to solving the stationary nonlinear problem (54) is to treat the (formally) non-stationary problem (45) (respectively the discrete counterpart (48)), using $t_{n+1}=t_{n}+\Delta t_{n}$ as an iteration parameter. Stationary solutions of the flow solver are computed in this way by initializing the flow field and marching forward in time. As soon as a specified accuracy criterion is met a grid adaptation is performed and time integration is resumed.

As accuracy criteria we use residuals or defects. More precisely, in connection with the flow solver, by residual we mean the following measure to quantify steadiness of the flow field. For this purpose we sum for each cell of the mesh the absolute value of the numerical fluxes at the cell interfaces, i.e., the numerical flux balance, corresponding to the continuity equation and weight it by the ratio of the cell size and the computational domain. Finally this quantity is scaled by the 
residual of the first time step on the actual grid. Hence, the residual is a relative accuracy measure and is referred to as normalized averaged (density) residual.

The accuracy of the approximate porous medium solution is also quantified in terms of a residual. It is in this case the $\ell_{2}$-norm of the defect vector obtained when plugging the solution into the discrete system of equations divided by the square root of the number of equations. In which sense "residual" is used will be always clear from the context.

Since we are only interested in steady state solutions, stationary solutions at the current iteration step are computed alternating between both solvers. Therefore, the iteration process can be summarized as follows:

Step 1: Initialize flow solver.

Step 2: Transfer data $\left(T_{H G}, \mathbf{V}_{H G}\right)$ provided by flow solver to the porous medium solver.

Step 3: Converge porous medium solver until the residual is smaller than a given tolerance.

Step 4: Transfer data $\left(\left.\rho_{f}\right|_{\Gamma_{H G}}, T_{s} \mid \Gamma_{H G}\right)$ from porous medium solver to flow solver.

Step 5: Converge flow solver until the residual is smaller than a given tolerance.

Step 6: Perform grid adaptation in the flow solver.

Step 7: Return to step 2 until (55) is fulfilled.

The relevant parameters and tolerances in the algorithm will be specified later below.

\section{RESULTS}

Simulations of coolant injection through porous material are carried out based on the above coupling strategies. The results are compared with results from hot gas channel experiments. We begin with briefly describing the setup of the experiments conducted by Langener et al. [16]. Afterwards, the numerical setup is discussed. Since the experiments have been performed for a symmetric configuration and the turbulent flow is modeled using the RANS equations, we present corresponding two-dimensional simulations. Thus, influences from the sidewalls of the channel are neglected. To take these effects into account, three-dimensional simulations are subsequently presented as well.

\subsection{Experimental setup}

In Langener et al. [16] experiments are carried out using carbon/carbon $(\mathrm{C} / \mathrm{C})$ ceramics as porous material. $\mathrm{C} / \mathrm{C}$ material is a ceramic matrix composite (CMC) where both fibers and matrix are made from carbon. Temperatures are measured at locations in the material at different depths. For the experiments, the porous material is mounted into the sidewall of a subsonic wind tunnel. On the backside of the $\mathrm{C} / \mathrm{C}$ material a coolant reservoir is attached. The experimental setup is shown in Fig. 3. The test section is $1.32 \mathrm{~m}$ long with the $\mathrm{C} / \mathrm{C}$ material beginning $0.58 \mathrm{~m}$ downstream from the entrance, the height is $90 \mathrm{~mm}$ and the width $60 \mathrm{~mm}$. The $\mathrm{C} / \mathrm{C}$ probe measures $61 \mathrm{~mm} \times 61 \mathrm{~mm}$ and is $15 \mathrm{~mm}$ thick.

In the hot gas channel, the pressure is measured on one of the channel sidewalls, as can be seen in Fig. 3(a). The temperature distribution on the surface of the porous material is monitored by infrared thermography. In addition the temperature in the porous material at different depths is measured by thermocouples. So far it is not clear whether these measurements are influenced by 


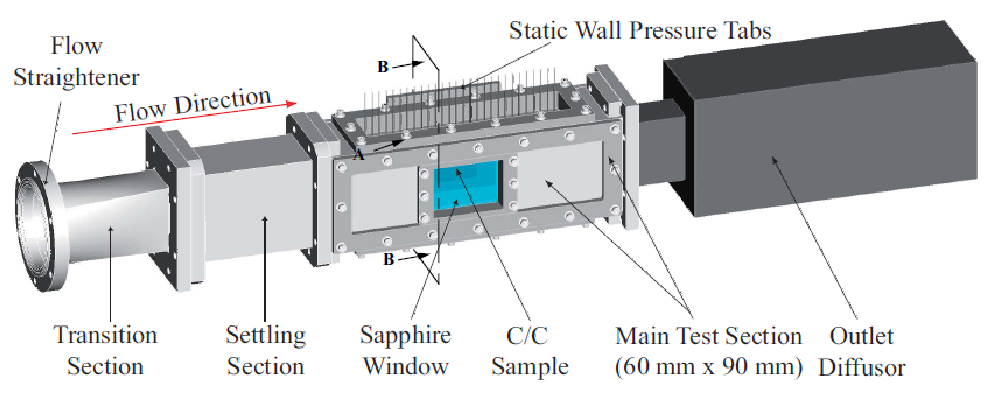

(a) Hot gas channel

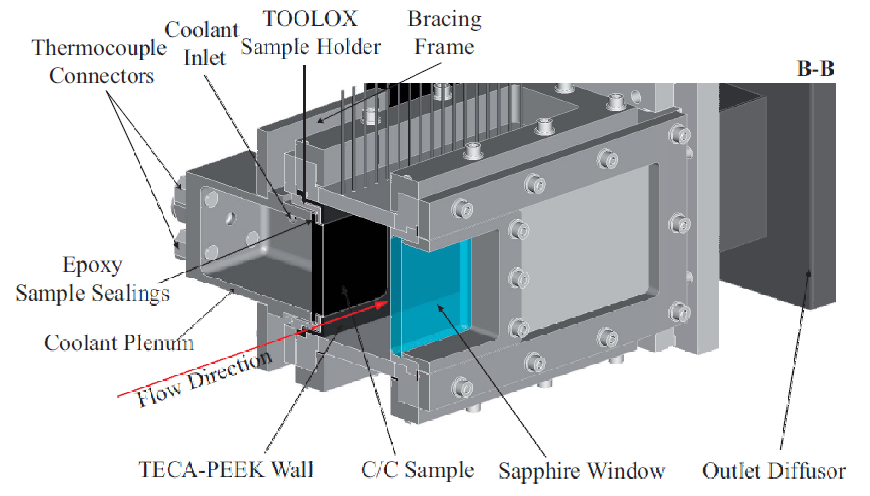

(b) Sample integration

Figure 3. Experimental setup by Langener et al. [16].

the coolant. Hence, it is assumed that they represent the temperature of the solid. The condition of the coolant in the reservoir is monitored as well. The experiments are described in detail in Langener et al. [16]. The turbulent flow conditions in the hot gas channel are summarized in Tab. I. The boundary layer in the channel has been estimated using pitot elements. The boundary layer thickness is about $\delta=20 \mathrm{~mm}$. Since in spanwise direction there are only $14.5 \mathrm{~mm}$ left from the porous material to the sidewalls of the channel they are expected to influence the coolant injection.

The parameters concerning the $\mathrm{C} / \mathrm{C}$ material and the coolant (air) are listed in Tab. II and III, respectively. The C/C material is produced by the DLR Stuttgart [17]. The probe used in the experiments corresponding to the simulations presented here is mounted into the wall in such a way that the flow through the material is orthogonal to the direction of the ceramic fibers. Possible differences to be expected when mounting the porous material for parallel throughflow will be discussed in the conclusion.

With these data it is not possible to obtain quantitative comparisons between $\mathrm{C} / \mathrm{C}$ materials with orthogonal and parallel flow directions. Experiments with probes coming from the same production batch are planned and corresponding numerical simulations will be performed afterwards. Still, such probes for orthogonal and parallel throughflow will not have the same material parameters since additional production processes are necessary to produce the latter.

\subsection{Numerical setup $(2 D)$}

Exploiting symmetry we begin two-dimensional simulations of the transpiration cooling problem. To that end, we apply the weak coupling of the finite volume solver Quadflow with the finite element 


\begin{tabular}{lll}
\hline Mach number & $M a$ & 0.5 \\
density & $\rho_{H G}$ & $0.65 \mathrm{~kg} / \mathrm{m}^{3}$ \\
total temperature & $T_{t, H G}$ & $525 \mathrm{~K}$ \\
pressure & $p_{H G}$ & $95600 \mathrm{~Pa}$ \\
\hline
\end{tabular}

Table I. List of flow conditions in the hot gas channel.

\begin{tabular}{lll}
\hline throughflow direction & & orthogonal \\
porosity & $\varphi$ & 0.116 \\
density & $\rho_{s}$ & $1.14 \mathrm{~kg} / \mathrm{m}^{3}$ \\
spec. heat capacity & $c_{p, s}$ & $622 \mathrm{~J} /(\mathrm{kgK})$ \\
eff. heat conductivity & $k_{s}$ & $1.4 \mathrm{~W} /(\mathrm{mK})$ \\
permeability & $K_{D}$ & $1.19610^{-13} \mathrm{~m}^{2}$ \\
Forchheimer coeff. & $K_{F}$ & $8.810^{-9} 1 / \mathrm{m}$ \\
\hline \multicolumn{2}{c}{ Table II. List of porous media parameters. }
\end{tabular}

Table II. List of porous media parameters.

\begin{tabular}{llc}
\hline spec. heat capacity & $c_{p, f}$ & $1010 \mathrm{~J} /(\mathrm{kgK})$ \\
eff. heat conductivity & $k_{f}$ & $0.04 \mathrm{~W} /(\mathrm{mK})$ \\
dynamic viscosity & $\mu$ & $1.710^{-5} \mathrm{Ns} / \mathrm{m}^{2}$ \\
\hline \multicolumn{2}{c}{ Table III. List of coolant parameters (air). }
\end{tabular}

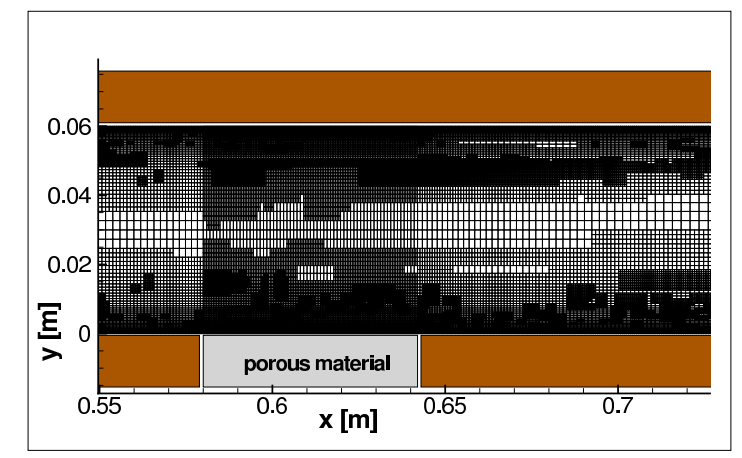

Figure 4. Detail of final adaptive grid.

porous media solver on the boundary $\Gamma_{H G}$ described in Section 5. On the hot gas side, the twodimensional turbulent flow through a channel is modeled using the Menter SST turbulence model.

The coarse grid for the flow solver comprises 190 grid cells and 5 refinement levels are used. The grid lines are concentrated towards the wall using a stretching function. The final adaptive grid consists of about 40.000 grid cells, see Fig. 4 .

As mentioned earlier, at the inflow boundary $\Gamma_{I}$, using the law of the wall, a turbulent profile for temperature and velocity is prescribed. At the outflow boundary $\Gamma_{O}$ only the pressure is prescribed. The channel walls are adiabatic and no-slip boundary conditions are imposed.

The channel walls on both sides of the porous material are adiabatic walls. The values for the porous media boundary conditions are summarized in Tab. IV. Concerning these test cases, the Darcy velocities in the porous material are rather small. Therefore, it is not necessary to stabilize the elliptic part (28) (see (32)) of the finite element porous medium solver. For computing a stationary 


\begin{tabular}{llllll}
\hline blowing ratio & $F$ & 0.001 & 0.002 & 0.003 & 0.01 \\
coolant reservoir pres. & $p_{c}$ & $326400 \mathrm{~Pa}$ & $448400 \mathrm{~Pa}$ & $544400 \mathrm{~Pa}$ & $1093400 \mathrm{~Pa}$ \\
coolant reservoir temp. & $T_{c}$ & $336.3 \mathrm{~K}$ & $319.1 \mathrm{~K}$ & $311.7 \mathrm{~K}$ & $296.6 \mathrm{~K}$ \\
solid temp., reservoir & $T_{s, R}$ & $403.3 \mathrm{~K}$ & $363.4 \mathrm{~K}$ & $342.6 \mathrm{~K}$ & $304.1 \mathrm{~K}$ \\
\hline
\end{tabular}

Table IV. List of porous media boundary conditions.

solution with the flow solver, an implicit backward Euler time integration scheme is used with local time steps determined by a global CFL number. The following CFL evolution strategy

$$
C F L_{n+1}=\min \left(C F L_{\min } \cdot 1.05^{n}, C F L_{\max }\right)
$$

is used with the parameters $C F L_{\min }=1$ and $C F L_{\max }=100$. Here the index $n$ enumerates the number of time steps since the last grid adaptation, i.e., after each adaptation the CFL number is again set to $C F L_{\min }$. This is essential because each grid adaptation causes a perturbation of the steady-state solution corresponding to the old grid thereby triggering some instationary waves on the new grid. When approaching the steady state on a current grid larger CFL numbers are admissible.

The porous material is discretized by an equidistant coarse grid with $8 \times 2$ degrees of freedom which is uniformly refined up to a given maximal resolution level $l$. That is, because of the uniform porosity, no local grid adaptation is performed in the porous medium domain. Both the linear system for the temperature equations and the linearized system for the momentum and continuity equations are solved by employing the direct solver UMFPack. During the Picard iteration the residual of the transport system is reduced in about four iterations by seven orders of magnitude. Four such outer iterations in the porous medium flow solver are performed for dropping the residual of the complete system below $10^{-10}$.

In the course of the computation grid adaptation is applied in the hot gas flow whenever the normalized averaged density residual has dropped by three orders of magnitude. After each grid adaptation in the hot gas flow we transfer data from the output of the flow solver to the porous medium solver and then apply the porous medium solver, i.e., we execute a coupling iteration to update the temperature of the fluid and the mass flow of the coolant at the interface $\Gamma_{H G}$. This is done for a prescribed number $m$ of coupling iteration steps to be specified below.

After the last coupling iteration the computation in the hot gas flow continues until a residual drop of $10^{-5}$ is reached. The highest level $L$ of grids used for the hot gas flow simulation is kept fixed. Since in each grid-adaptation step an additional refinement level can be introduced and the computation is started on level $L=1$, the final grid level $L$ could be already reached after $L-1$ grid adaptations. Note that the preceding adaptation steps are only used to generate an initial guess for the iteration on the final grid level $L$. Therefore these runs do not have to be fully converged in time.

A complete theoretical justification of the various process parameters is hardly feasible. Therefore, preparatory computational studies are performed for various parameter choices and modes of operations. Subsequent simulations are then to employ a parameter choice which on the one hand is to keep computational complexity moderate while, on the other hand, in a test scenario presumably more accurate but also more expensive choices do not show any significant deviations. In order to justify the choices of 
$L$ the highest grid level generated in the course of adaptation steps in the hot gas flow,

$l$ the number of uniform grid refinements in the porous medium flow,

$m$ the number of coupling iteration steps in the simulation of the hot gas flow, we conduct first a parameter study for the exemplarily blowing ratio $F=0.01$. In Fig. 5(a), grid convergence for the hot gas domain is verified by showing the wall temperature for different grid refinement levels. For $L=5$, there is a small difference in comparison with higher levels. Since the results are essentially indistinguishable for the higher levels, $L=6$ is taken for all subsequent computations.

Different grids are investigated for the porous material as well, see Fig. 5(c). Since the mass flux of coolant transported through the porous material is a key variable in this investigation we present the velocity component $v_{y}$ of $\mathbf{V}$ in porous media flow orthogonal to $\Gamma_{H G}$. For $l=3$ and $l=4$, the resolution appears to be too coarse and the results differ significantly from those for higher refinement levels. For levels equal or higher than $l=5$, there is no visible difference anymore.

To investigate the influence of the coupling process the wall temperature $T_{N S}$ in the hot gas channel is displayed for different numbers of coupling iteration steps $m$ in Fig. 5(b). The highest grid level $L=6$ is reached during coupling step $m=5$. There are only small differences for iteration step $m=6$ but no detectable further deviations for additional coupling steps. Concerning the porous medium flow, we discuss only the temperature $T_{f}$ of the coolant because there are much smaller variations in the velocity component $v_{y}$, see Fig. 5(c). In contrast to the hot gas flow, the difference in $T_{f}$ between iteration steps $m=6$ and $m=7$ is larger, see Fig. 5(d). After the fifth grid adaptation in the hot gas flow the porous medium computation is performed before the solution in the hot gas flow field could adapt to the new grid involving the final level $L=6$. In the subsequent iteration steps $m=7, \ldots, 10$, while the hot gas flow solution is computed without further increasing the grid level, the solutions in the porous material show no further deviations.

Aside from the temperature distributions the relevant quantity for the coupling process is the pressure difference at $\Gamma_{H G}$ which we discuss now. In Fig. 6 we show the relative pressure, i.e., the pressure scaled by the free stream pressure of the hot gas, for both the porous medium and the hot gas at the interface for different coupling iteration steps. In Table $\mathrm{V}$ we list the corresponding $l_{1}$-norm of the pressure difference at the interface $\Gamma_{H G}$ relative to the free stream pressure $p_{\infty}$ of the hot gas flow, see (55). We note that the pressure in the hot gas does not change significantly. However, there is a significant change in the pressure of the porous medium flow that gradually approaches the pressure of the hot gas with each further coupling iteration step. Obviously, $m=8$ coupling iteration steps are sufficient to provide a sufficient agreement between the pressures at the interface. Therefore we fix $m=8$ for our simulations.

On account of these findings, the subsequent computations use $L=6$ and $l=5$ refinement levels for the flow solver and the porous medium solver, respectively. Eight coupling iteration steps, i.e., $m=8$, are carried out where the next coupling iteration is triggered whenever the normalized, averaged density residual of the flow solver has dropeed below $10^{-3}$. As indicated before, the coupling step consists in a call of the porous medium solver using data provided by the hot gas flow solver. After the last iteration, the flow solver is converged until a normalized, averaged density residual of at least $10^{-5}$ is reached, using the updated boundary conditions provided by the porous medium solver. . 

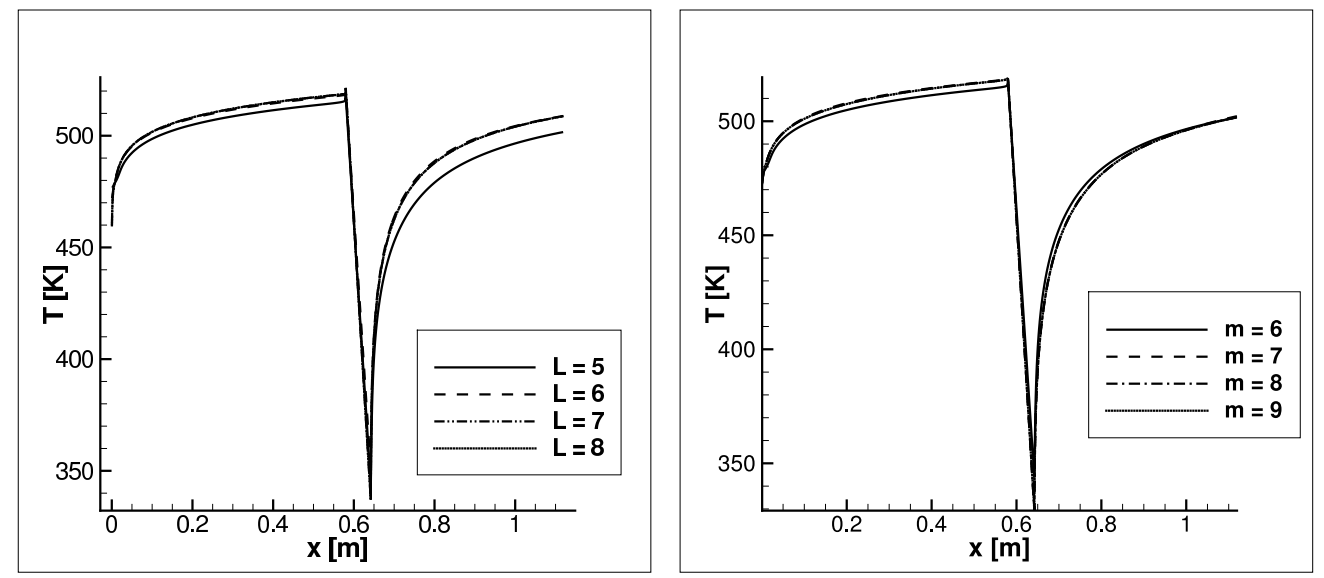

(a) Hot gas wall temperature for different grid (b) Hot gas wall temperature for different number of refinement levels $L$, fixed number of coupling iteration coupling iteration steps $m$, fixed grid refinement levels steps $m=8$ and porous media grid level $l=5$.

$$
L=6 \text { and } l=5 \text {. }
$$
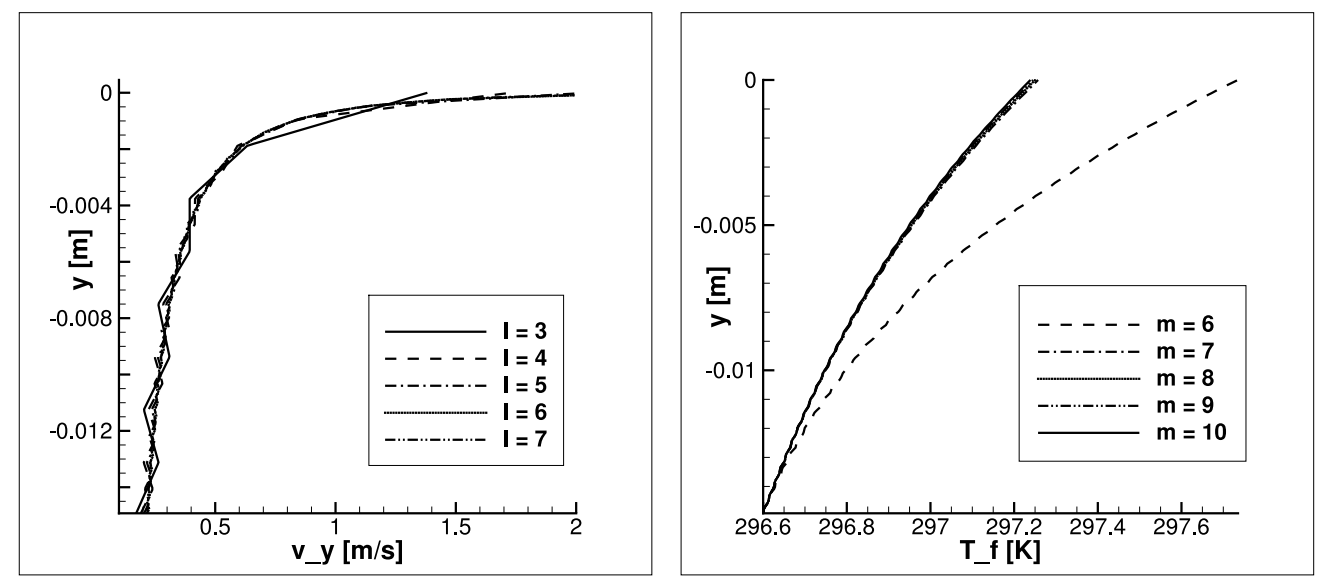

(c) Velocity component $v_{y}$ in the porous material for (d) Coolant temperature in the porous material for different grid refinement levels $l$, fixed number of different number of coupling iteration steps $m$, fixed coupling iteration steps $m=8$, and hot gas grid level grid refinement levels $L=6$ and $l=5$.

$L=6$.

Figure 5. Convergence studies.

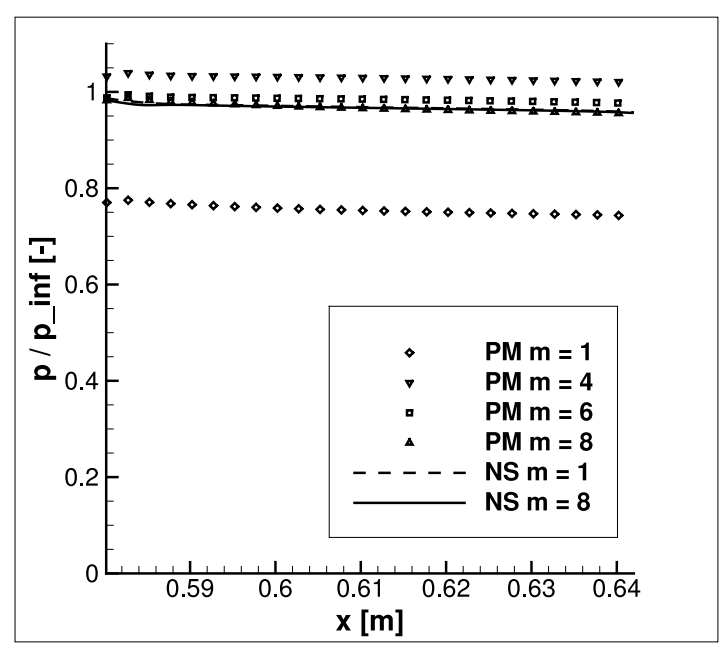

Figure 6. Pressure at interface. 


\begin{tabular}{c|c}
$m$ & $\left\|\left(\left.p_{N S}\right|_{\Gamma_{H G}}-\left.p_{P M}\right|_{\Gamma_{H G}}\right)\right\|_{l_{1}} / p_{\infty}$ \\
\hline 1 & 0.013747 \\
2 & 0.038956 \\
3 & 0.006312 \\
4 & 0.003054 \\
5 & 0.001350 \\
6 & 0.000713 \\
7 & 0.000483 \\
8 & 0.000281
\end{tabular}

Table V. Relative pressure difference at the interface $\Gamma_{H G}$ for different coupling iteration steps $m$.

\subsection{Numerical results $(2 D)$}

In this section, we present first the distribution of several quantities in the hot gas flow and in the porous material provided by the two-dimensional simulations for different blowing ratios. This is followed by comparing the results obtained by the two-dimensional finite element porous medium solver with results from a one-dimensional finite difference solver, cf. Gerber [10], and experimental data, cf. Langener et al. [16]. The limitations of both, numerics and measurement technologies are discussed.

The temperature in the hot gas flow in the region of the porous material injection is shown in Fig. 7. Upstream the injection, a normal temperature profile for an adiabatic channel flow can be seen, with a maximum temperature on the centerline of the channel, a minimum temperature in the boundary layer and a slightly increased wall temperature. A coolant film is now created by the injection through the porous material with increasing thickness over the length of the probe. With increasing blowing ratio $F$, the thickness of the coolant film increases as well. Furthermore, its temperature decreases, due to less heat consumption per unit mass flow inside the porous material which again results from a higher coolant mass flux. Further downstream the injection the temperature in the coolant layer increases, mainly due to turbulent mixing.

Fig. 8 shows the wall-normal momentum of the injection. For lower blowing ratios only a small disturbance of the channel flow can be seen. For higher blowing ratios a mushroom like shape forms. For $F=0.01$ a strong effect from the injection on more than half the channel height can be observed. Especially above the leading edge of the porous material at $x=0.58 \mathrm{~m}$, a high momentum appears. This results from the injection being an obstacle for the hot gas flow. The latter has to detach from the wall and rise above the injection, leading to a high wall-normal momentum.

The disturbance introduced by the injection into the hot gas flow leads to a production of turbulence. The turbulent kinetic energy is shown in Fig. 9. For the highest blowing ratio $F=0.01$ the streak of turbulent kinetic energy beginning at the leading edge of the porous material is clearly visible. For lower blowing ratios and therefore less wall-normal momentum and less disturbance of the hot gas flow, the production of turbulent kinetic energy is much smaller.

In the following we present the condition of both fluid and solid in the porous medium beginning with the temperature of the solid in Fig. 10. At the bottom close to the coolant reservoir the solid is rather cold. With increasing blowing ratio the temperature decreases because of the higher cooling potential of the larger coolant mass flux and the coolant, being replaced more frequently, stays cooler inside the reservoir. On the hot gas side the solid is hotter at the leading edge of the porous 


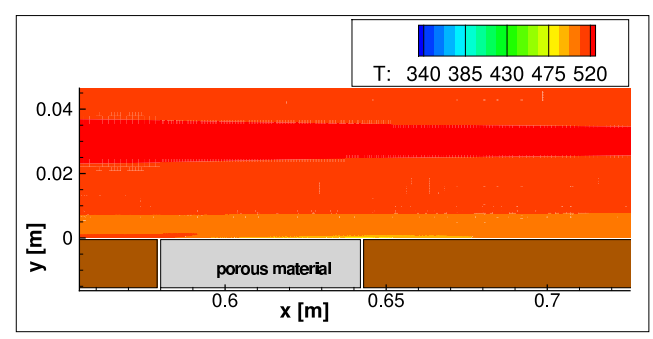

(a) $F=0.001$

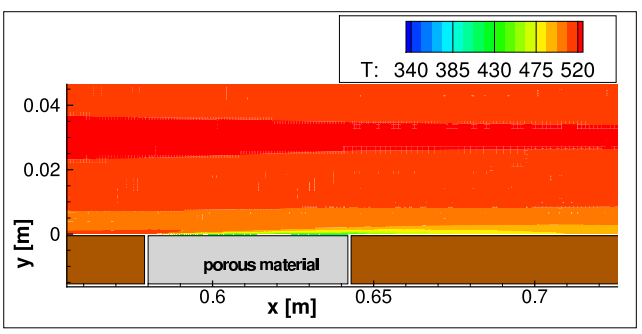

(c) $F=0.003$

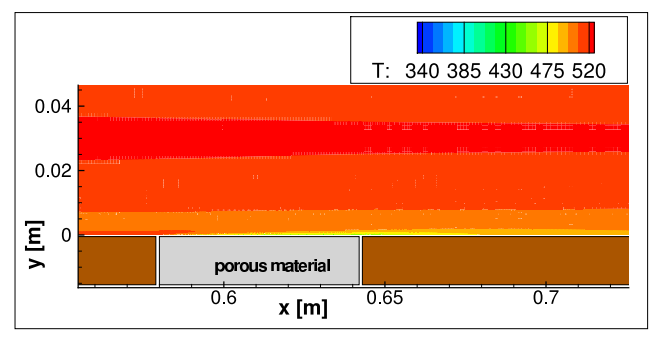

(b) $F=0.002$

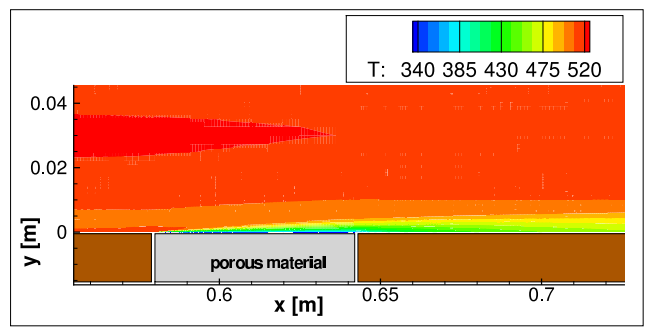

(d) $F=0.01$

Figure 7. Temperature distribution in hot gas flow for different blowing ratios.

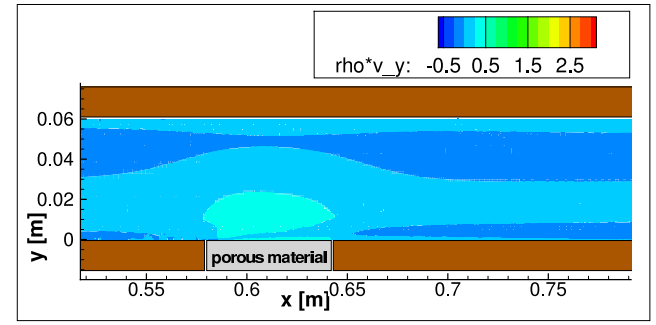

(a) $F=0.001$

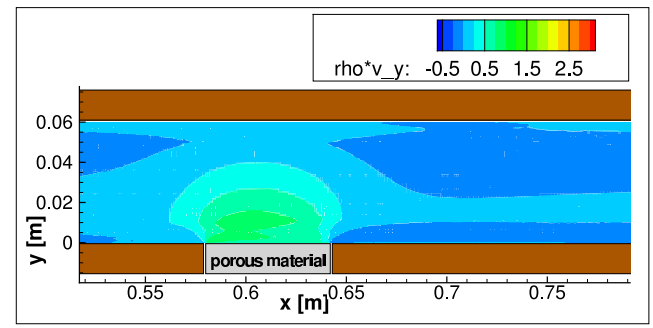

(c) $F=0.003$

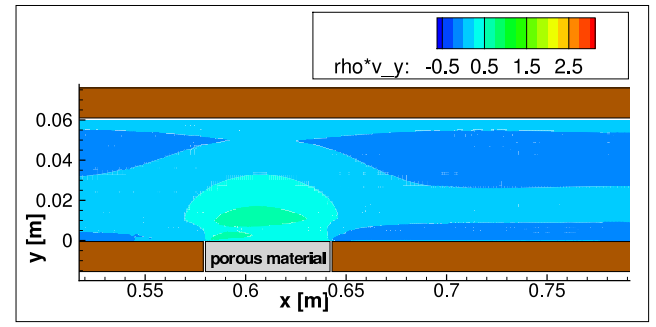

(b) $F=0.002$

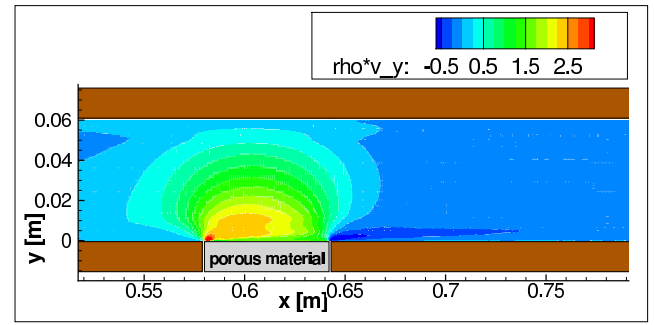

(d) $F=0.01$

Figure 8. Wall normal momentum in hot gas flow for different blowing ratios.

material and cooler further downstream, because of the developing coolant film in the hot gas flow. This effect again increases with increasing blowing ratio since, due to the higher coolant mass flux, the coolant film develops more rapidly. The difference between the temperature of the solid close to the coolant reservoir and close to the leading edge of the porous material increases with increasing blowing ratio because the temperature of the hot gas flow differs less at this point compared with the temperature close to the reservoir.

The temperature of the coolant inside the porous material, see Fig. 11, is strongly coupled with the temperature of the solid and therefore shows essentially the same behavior. In contrast to the temperature of the solid, due to higher Darcy velocities, the temperature difference decreases with 


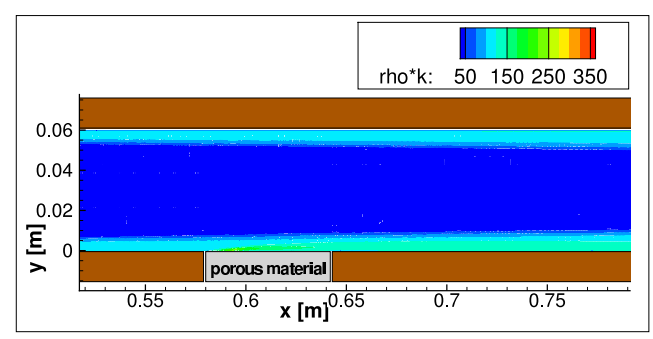

(a) $F=0.001$

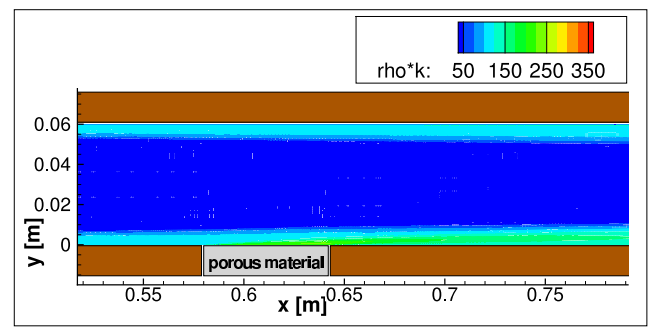

(c) $F=0.003$

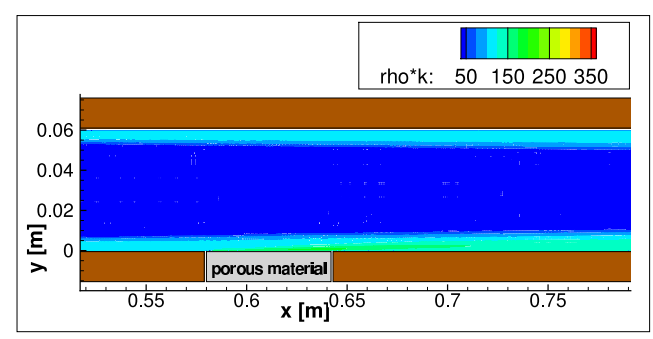

(b) $F=0.002$

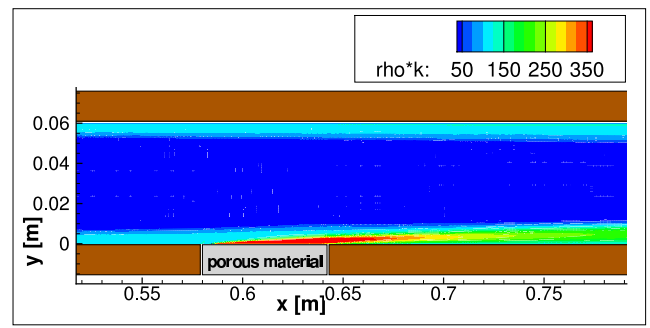

(d) $F=0.01$

Figure 9. Turbulent kinetic energy in hot gas flow for different blowing ratios.

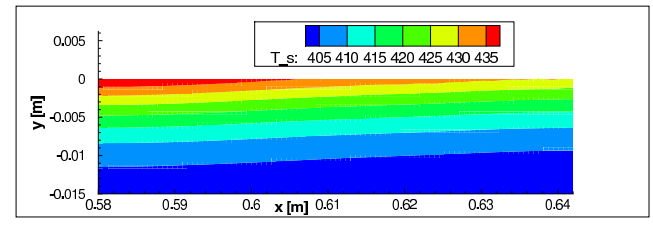

(a) $F=0.001$

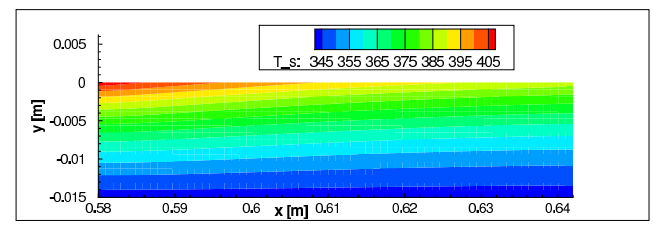

(c) $F=0.003$

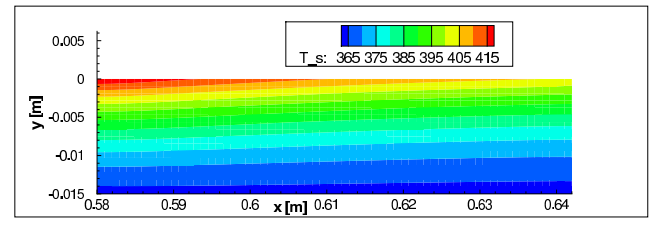

(b) $F=0.002$

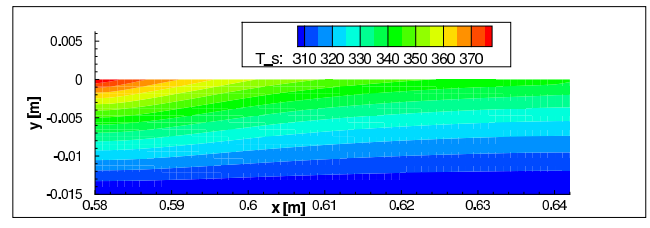

(d) $F=0.01$

Figure 10. Temperature of solid in porous material for different blowing ratios.

increasing blowing ratio. Therefore, the through-flow time of the coolant is smaller and less heat is consumed by each portion of the coolant.

Fig. 12 displays the density of the fluid inside the porous material. Due to higher pressure in the coolant reservoir and smaller pressure in the hot gas flow, the density evolves from higher values in the coolant reservoir to lower values on the hot gas side. Since a higher pressure is necessary for higher blowing ratios, the density is increasing with $F$ as well. There are no significant changes according to the run length $x$ of the hot gas flow, the results are quasi one-dimensional.

The Darcy velocities resulting from the given pressure difference are shown in Fig. 13. The quantitative behavior is quite similar for the different test cases. According to the steeper pressure gradient close to the hot gas flow, the velocity increases. As expected, the velocity increases with increasing blowing ratio. Again, there is no noticeable dependence in direction of the hot gas flow even though, due to the injection, there is a pressure drop in the hot gas flow. But the overall pressure 


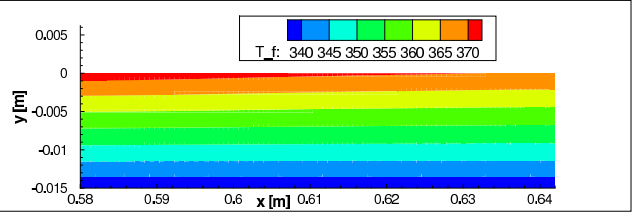

(a) $F=0.001$

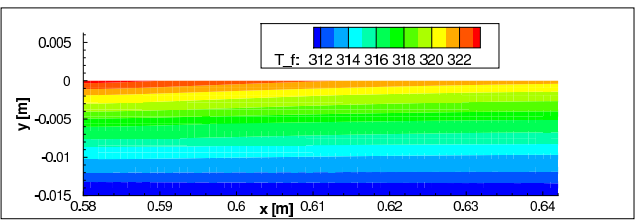

(c) $F=0.003$

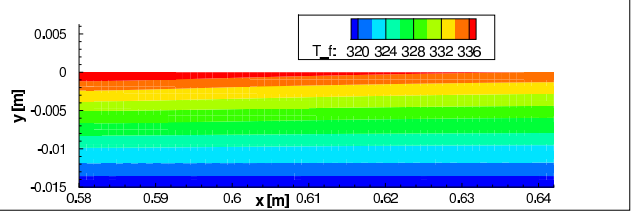

(b) $F=0.002$

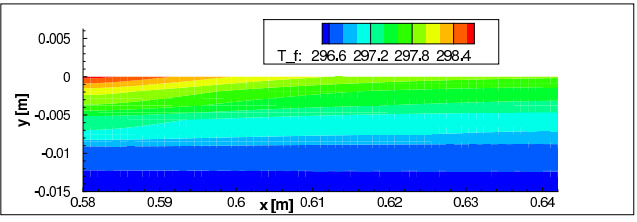

(d) $F=0.01$

Figure 11. Temperature of fluid in porous material for different blowing ratios.

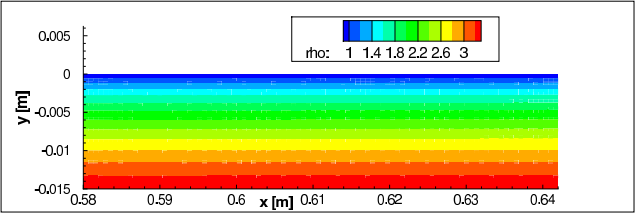

(a) $F=0.001$

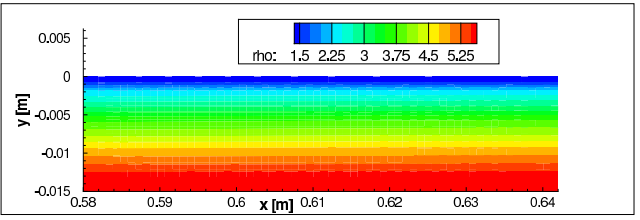

(c) $F=0.003$

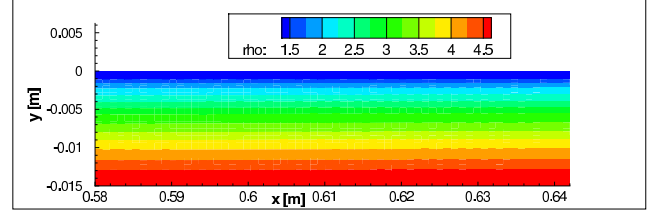

(b) $F=0.002$

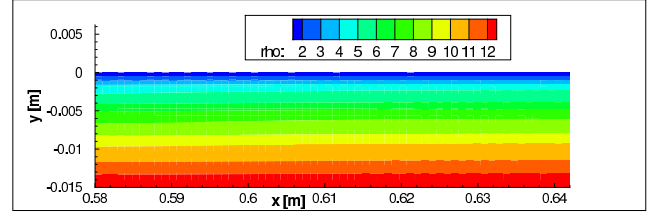

(d) $F=0.01$

Figure 12. Density of fluid in porous material for different blowing ratios.

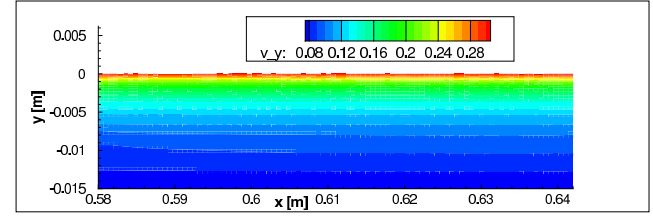

(a) $F=0.001$

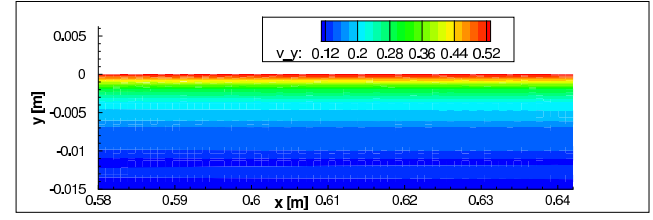

(c) $F=0.003$

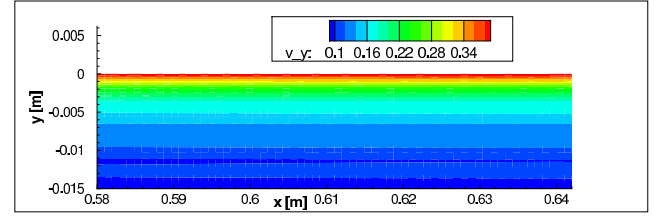

(b) $F=0.002$

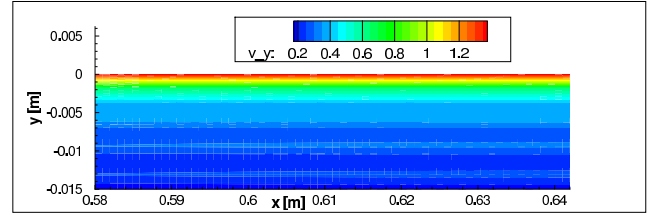

(d) $F=0.01$

Figure 13. Darcy velocity in porous material for different blowing ratios.

difference in the hot gas channel is small compared to the one in the porous medium that necessary to drive the coolant through the $\mathrm{C} / \mathrm{C}$ material.

Fig. 14 shows the cooling efficiency $\eta_{a d}$ for the different blowing ratios. Upstream the injection zone the cooling efficiency is zero or even slightly negative. The latter results from the deceleration of the hot gas flow due to the injection being an obstacle. The highest values are reached directly 


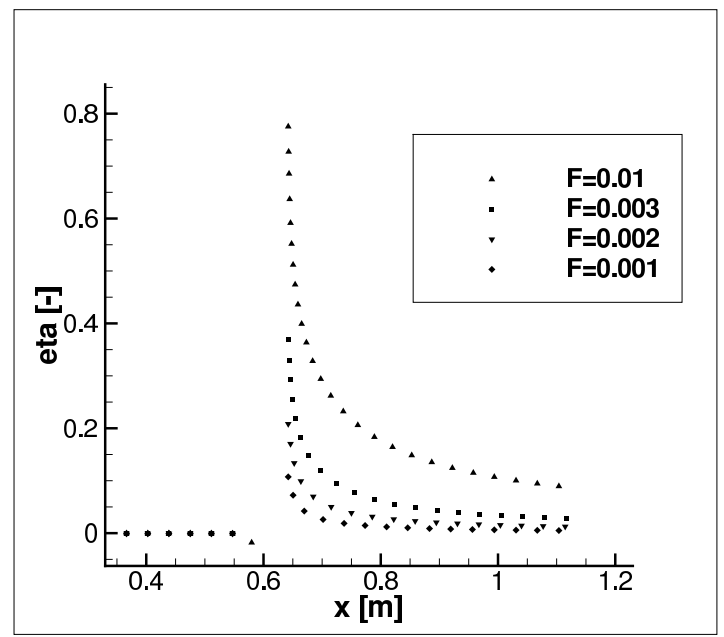

Figure 14. Cooling efficiency $\eta_{a d}$ for different blowing ratios.

downstream the porous material, reaching up to 80 percent for the highest blowing ratio. Further downstream, due to the turbulent mixing process, the cooling efficiency decreases quickly. At least for $F=0.01$, a substantial cooling efficiency lasts three lengths of the porous material downstream.

In Fig. 15, the temperature of the solid taken at different $y$ positions in the porous material is compared with results from a one-dimensional porous medium solver and experimental data. Langener et al. [16] placed thermocouples in different depths of the $\mathrm{C} / \mathrm{C}$ material. The onedimensional solver [10] uses averaged values at the coupling interface.

As already seen in Fig. 10 the temperature at the hot gas side decreases in streamwise direction of the hot gas flow. For all blowing ratios the results from the one-dimensional solver lie between the results from the left and the right position in the two-dimensional porous material. For $F=0.002$ and $F=0.003$, taking the measurement errors into account, the computational results are in reasonable agreement with the experimental data. For the smallest and largest blowing ratio the temperature is, however, significantly under- and overestimated, respectively. This probably results from the choice of the heat transfer coefficients $h$ and especially $h_{H G}$. Although the latter is modeled as a function of the blowing ratio the influence of the coolant mass flow on the heat transfer seems to be underestimated. Better approximations of the heat transfer coefficients are necessary.

Four thermocouples are placed at the surface of the porous material. All four are shown in the figures and therefore illustrate the variations in the measurements across the surface. The thermocouples at different depths in the $\mathrm{C} / \mathrm{C}$ material cannot be placed above each other but are also distributed over the whole cross section. As can be seen in Fig. 10, at least close to the hot gas flow, there is a strong variation of the temperature of the solid in the direction of the hot gas flow. Furthermore, on the coolant reservoir side the closest measurement to the interface is taken $6 \mathrm{~mm}$ inside the porous material. This value is used as a boundary condition for the numerical simulations. Therefore, the temperature at this point is too high in Fig. 15(b) - 15(d).

\subsection{Numerical setup $(3 D)$}

To investigate the influences of the sidewalls of the hot gas channel on the results, three-dimensional simulations of the porous media injection into the channel are performed. The three-dimensional 


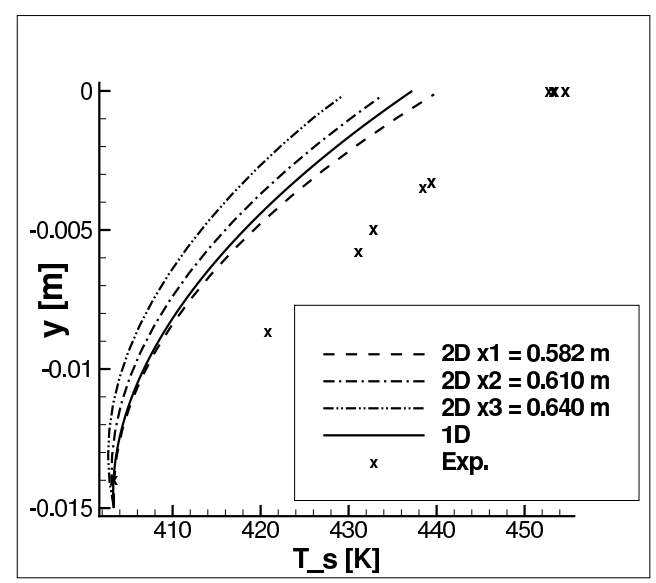

(a) $F=0.001$

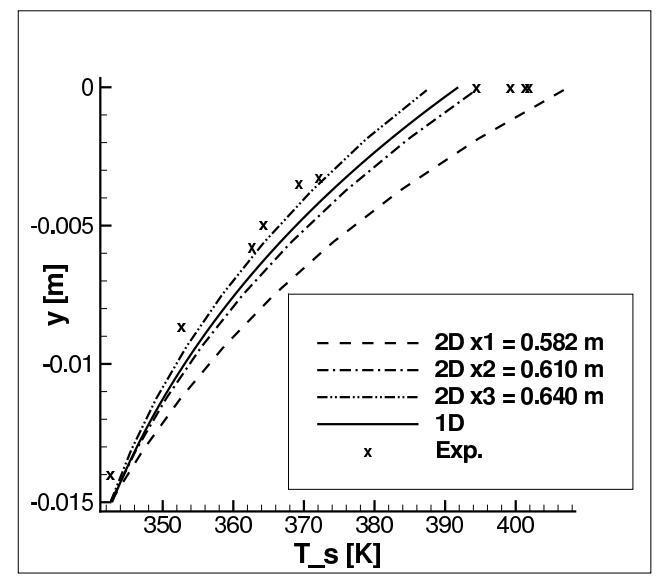

(c) $F=0.003$

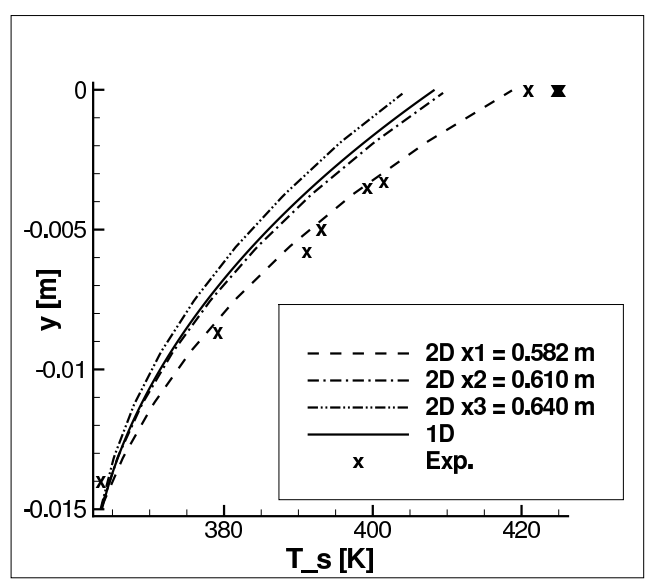

(b) $F=0.002$

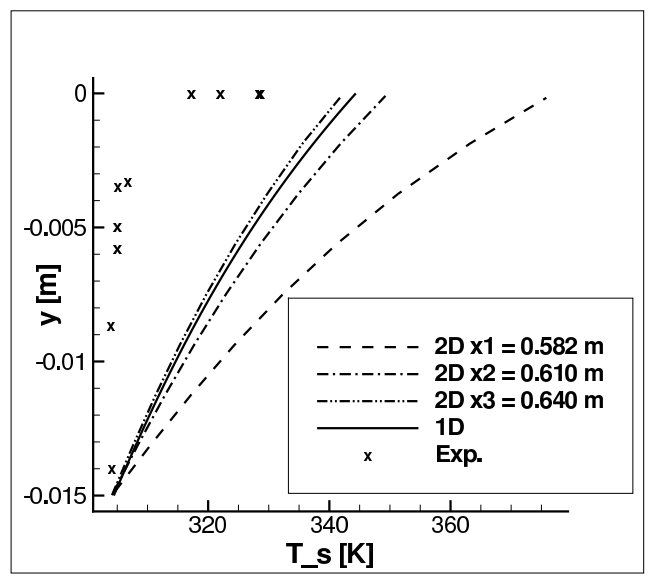

(d) $F=0.01$

Figure 15. Solid temperature in the porous material taken at $x_{1}=0.582 \mathrm{~m}, x_{2}=0.61 \mathrm{~m}$ and $x_{3}=0.64 \mathrm{~m}$ from two-dimensional simulations compared with one-dimensional simulations and experimental results.

grid is build starting from the two-dimensional configuration used before. Since the configuration is still axis-symmetric in spanwise direction, the grid is reduced to one half of the channel with symmetric boundary conditions on the centerline of the channel. The coarse grid is composed of 5824 grid cells. The number of grid cells in streamwise and wall-normal direction is doubled because of the small extent of the (half) channel in spanwise direction. A minimum number of 5 grid cells per block in each space dimension must be used for the coarse grid to guarantee the full functionality of the multiscale-based grid adaptation process. To compensate for this higher resolution of the coarse grid only three refinement levels are used. Note that due to these changes, the grid used here is not exactly a three-dimensional extension of the two-dimensional grid used before, but the grid spacing in streamwise and wall-normal direction is comparable. The final adaptive grid consists of about 8 million grid cells.

The porous medium is resolved by an equidistant grid with $32 \times 16 \times 32$ degrees of freedom. One can then proceed in a similar fashion as in the two-dimensional simulations. Specifically, we consider the blowing ratio $F=0.01$. Boundary conditions and material parameters, as summarized above for the two-dimensional setup, are taken here as well. 


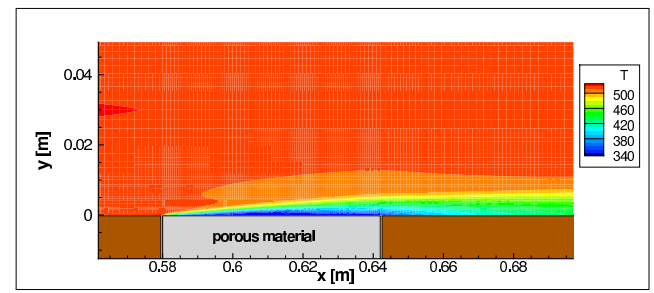

(a) $z=0.0 \mathrm{~m}$

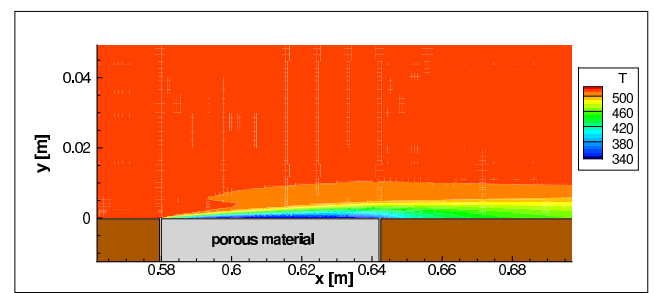

(b) $z=0.03 m$

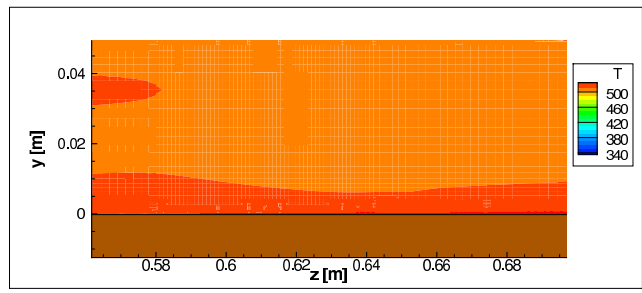

(c) $z=0.04 \mathrm{~m}$

Figure 16. Temperature distribution in hot gas channel at different spanwise positions.

\subsection{Numerical results $(3 D)$}

Since the hot gas channel in the experiment is quite narrow and the space between the porous material and the side walls of the channel is small the presence of walls are expected to influence the results.

First we show the temperature distribution in the hot gas channel at different spanwise positions in Fig. 16. For $z=0.0 m$, on the centerline of the channel, the coolant film develops as expected. Close to the edge in spanwise direction of the porous material the film becomes thinner, especially further downstream from the coolant injection, see Fig. 16(b). Alongside the porous material $(z=0.04 \mathrm{~m})$ no coolant film can be seen.

Considering the wall-normal momentum in Fig. 17, the solution on the centerline is again very similar to the two-dimensional result shown in Fig. 8(d). The injection leads to a mushroom-shaped form with a maximum wall-normal momentum close to the leading edge of the porous medium. Quantitatively, it is higher compared with the two-dimensional computation. At $z=0.03 m$, the momentum is much smaller. Besides the porous material $(z=0.04 \mathrm{~m})$, the momentum induced by the injection is still visible.

Comparing again the three-dimensional (Fig. 18) with the two-dimensional results (Fig. 9), the production of turbulent kinetic energy starts little further downstream, but still above the porous material. Furthermore, the absolute values are higher. Again, the effect reduces close to the edge of the porous material. There is no production of turbulence in between the edge of the porous material and the channel sidewalls.

Fig. 19 shows the temperature distribution at different streamwise positions, starting at the streamwise position of the injection. Furthermore, streamlines tangential to the slices which are normal to the main flow direction, are drawn. Therefore, these streamlines lack the streamwise velocity component. They must not be confused with common streamlines. In Fig. 19(a) the coolant injection can be seen at the bottom. Most of the injected coolant moves upwards, some is pushed sidewards into the gap between the injection regime and the sidewalls. Further downstream no more coolant is injected. Hot gas which has been displaced by the injection before cannot vanish, due 


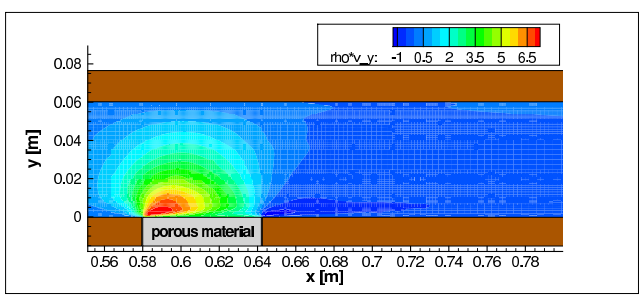

(a) $z=0.0 \mathrm{~m}$

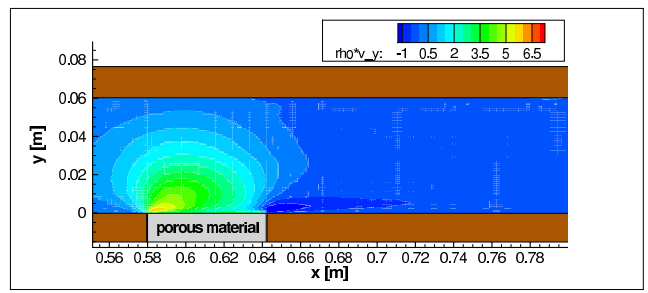

(b) $z=0.03 m$

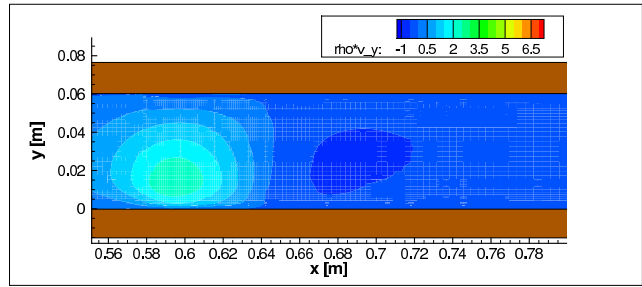

(c) $z=0.04 m$

Figure 17. Wall-normal momentum in hot gas channel at different spanwise positions.

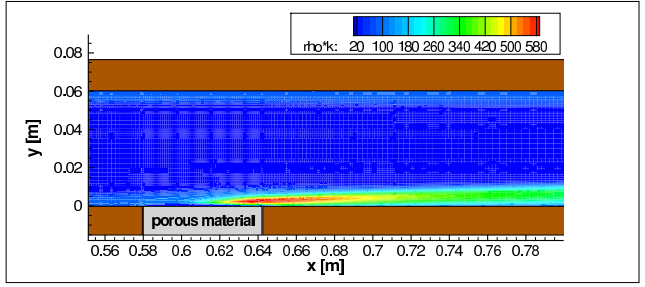

(a) $z=0.0 \mathrm{~m}$

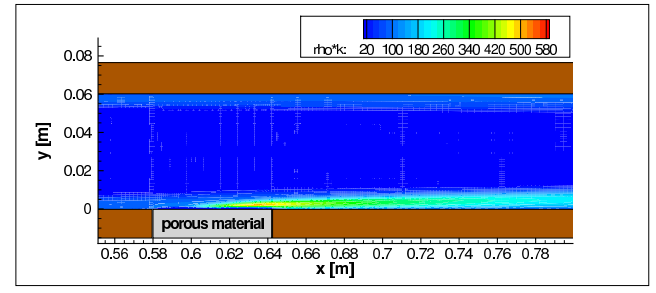

(b) $z=0.03 m$

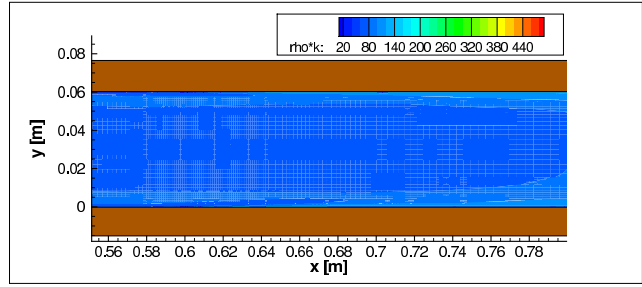

(c) $z=0.04 m$

Figure 18. Turbulent kinetic energy in hot gas channel at different spanwise positions.

to the channel sidewalls, but enters the cooling film from the side. The film becomes thicker and warmer, see Fig. 19(b)-19(d).

In the following the conditions inside the porous material are shown at different streamwise and spanwise positions. Starting with the temperature of the solid. In Fig. 20(a), the temperature on the centerline of the channel is shown which can be compared with the two-dimensional simulation, Fig. 10(d). The results match very well. Closer to the edge of the porous material, see Fig. 20(c), the temperature on the hot gas side of the interface is slightly increased, due to the hot gas coming from the non-cooled region on both sides of the porous material. Therefore, the temperature in the upper part of the porous material is increased as well.

Both streamwise slices close to the leading edge, see Fig. 20(b), and the trailing edge of the porous material, Fig. 20(d), confirm these observations. Near the centerline of the channel the temperature 


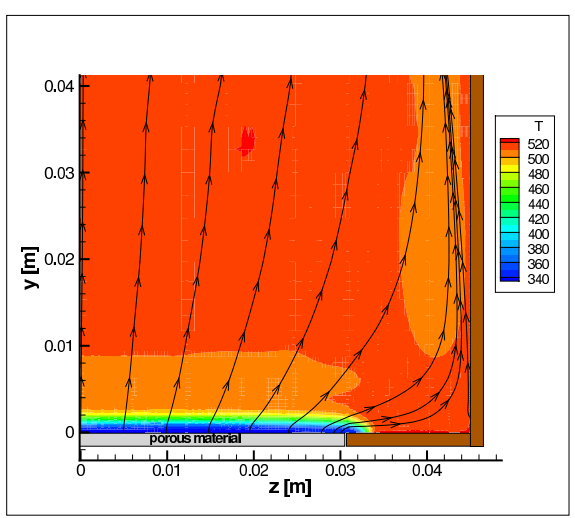

(a) $x=0.6 \mathrm{~m}$

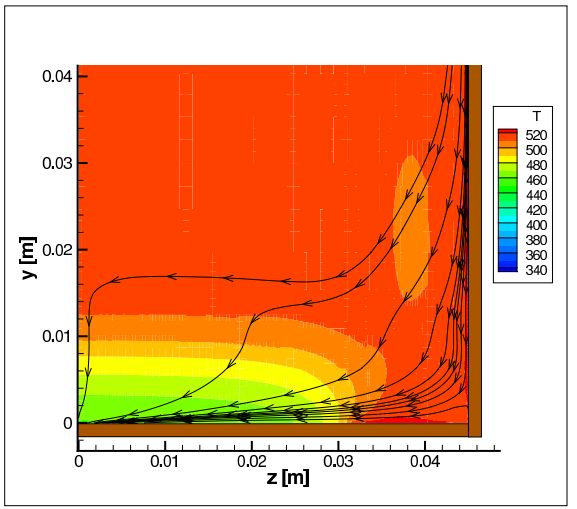

(c) $x=0.8 \mathrm{~m}$

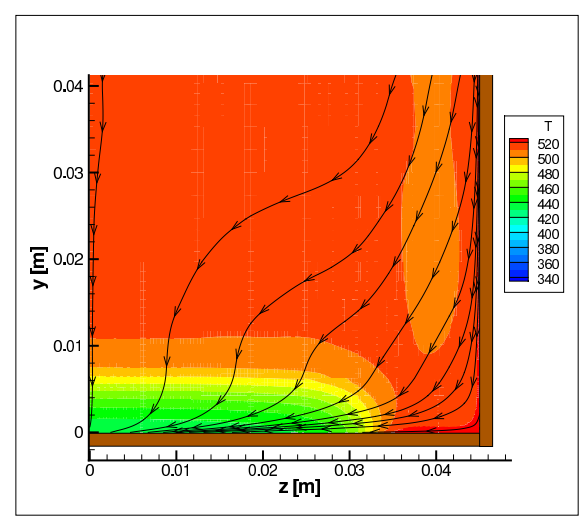

(b) $x=0.7 \mathrm{~m}$

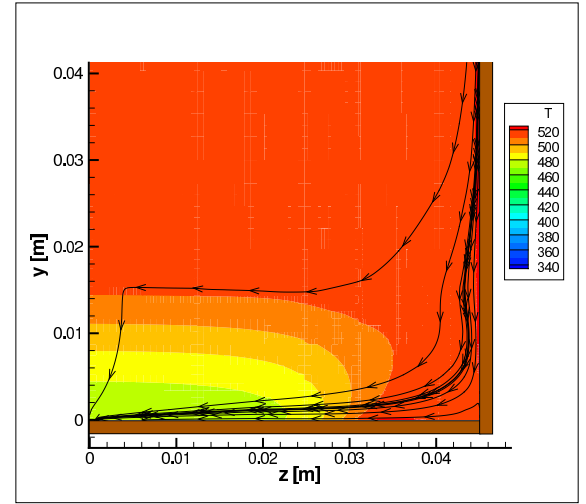

(d) $x=0.9 \mathrm{~m}$

Figure 19. Temperature distribution and tangential streamlines in hot gas channel at different streamwise positions.

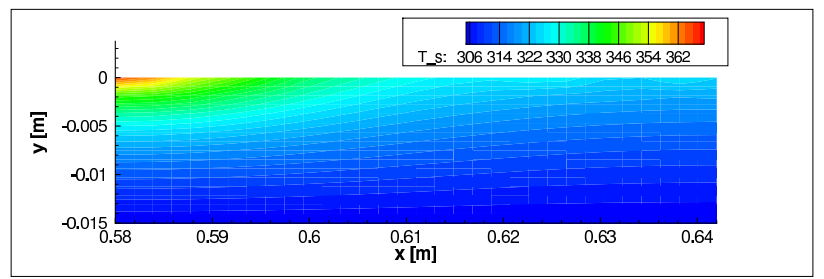

(a) $z=0.0 \mathrm{~m}$

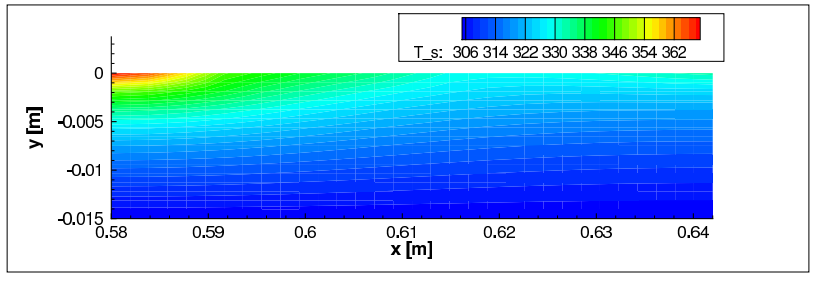

(c) $z=0.03 \mathrm{~m}$

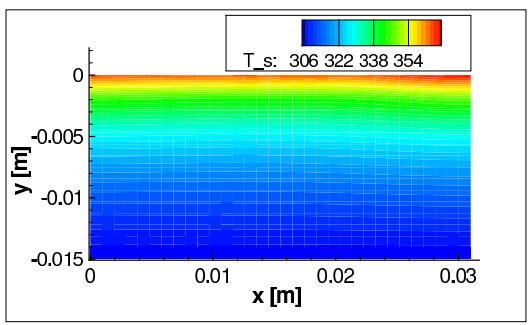

(b) $x=0.58 \mathrm{~m}$

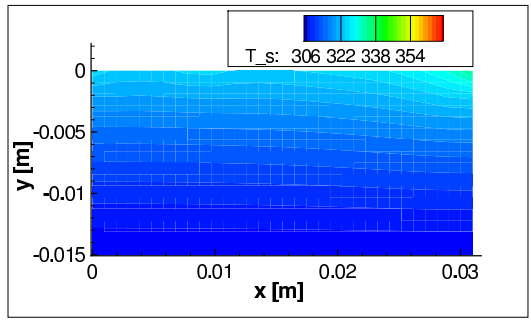

(d) $x=0.64 m$

Figure 20. Solid temperature in the porous medium at different spanwise ( (a) and (c) ) and streamwise ( (b) and (d) ) positions. 


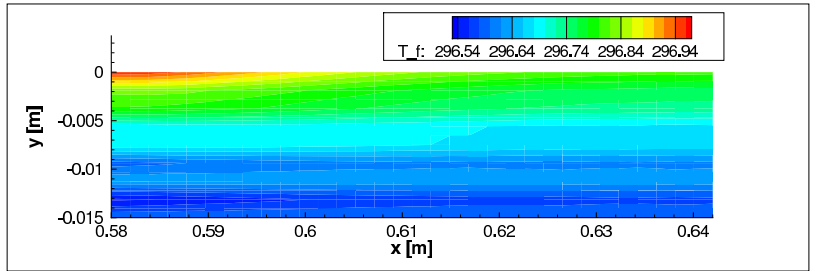

(a) $z=0.0 \mathrm{~m}$

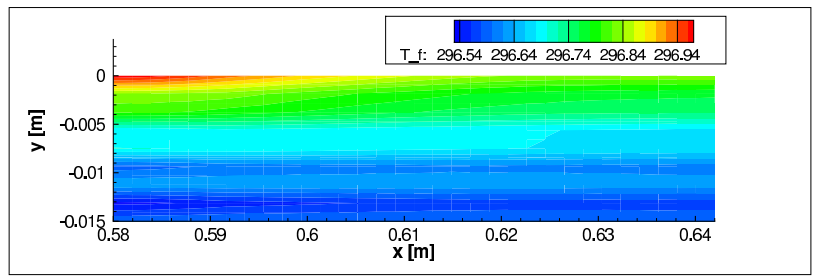

(c) $z=0.03 m$

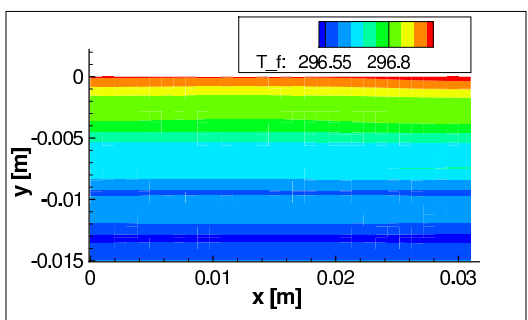

(b) $x=0.58 m$

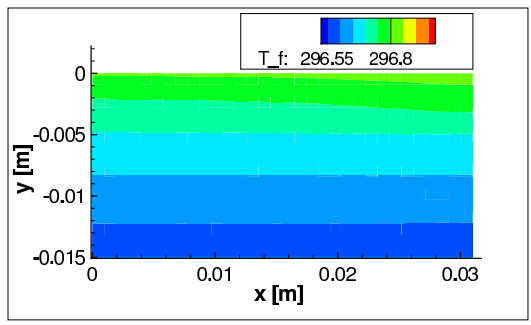

(d) $x=0.64 m$

Figure 21. Coolant temperature in the porous medium at different spanwise ( (a) and (c) ) and streamwise ( (b) and (d) ) positions.

distribution is two-dimensional. Closer to the edge in spanwise direction the temperature at the top increases.

The temperature of the coolant, see Fig. 21, behaves similarly, due to the coupling of the two temperature equations (4), (5) by the heat transfer term. The temperature reached close to the interface with the hot gas flow is lower compared to the two-dimensional simulation, Fig. 11(d). This is most likely due to a lower hot gas temperature. However, the difference is small in absolute values.

The coolant density does not show any variation in both spanwise and streamwise direction, see Fig. 22. Its distribution strongly depends on the given pressure difference between the coolant reservoir and the hot gas channel.

The cooling efficiency is shown in Fig. 23. There is no cooling effect upstream the leading edge of the porous material. There is no separation area in front of the injection as observed in supersonic film cooling simulations, see Dahmen et al. [9]. Therefore, here no coolant is moved upstream by a rotating vortex. Downstream the injection, there is also no cooling effect in the gap between the cooled region and the channel sidewall. Only directly on both sides of the porous material, a little coolant enters also the gap between the porous material and the channel sidewall. The highest cooling efficiency appears directly above the porous material. Further downstream it decreases. Furthermore, since the hot gas flow is sucked in from the side, as has already be seen in Fig. 19t, he cooled area is narrowed. Due to the absence of vortices or strongly rotating flow, there are no regions heated by hot gas transported directly to the wall, an effect observed by Dahmen et al. [9] when injecting coolant through a slot into a supersonic boundary layer.

The cooling efficiency is significantly higher on the centerline of the channel and decreases when approaching the edge of the porous material. This can be seen in Fig. 24 where the cooling efficiency in different spanwise positions is compared to the two-dimensional results. For $z=0.02 \mathrm{~m}$, the difference to the solution on the centerline is quite small. But close to the edge of the porous 


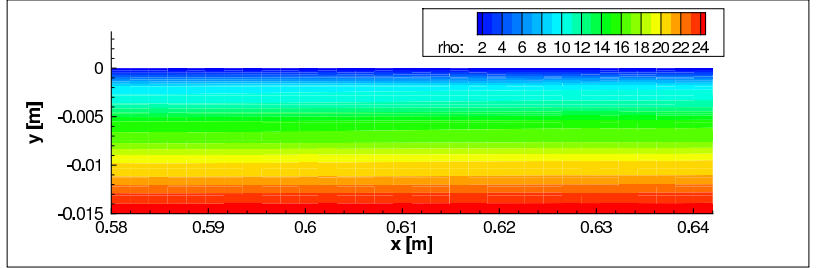

(a) $z=0.0 \mathrm{~m}$

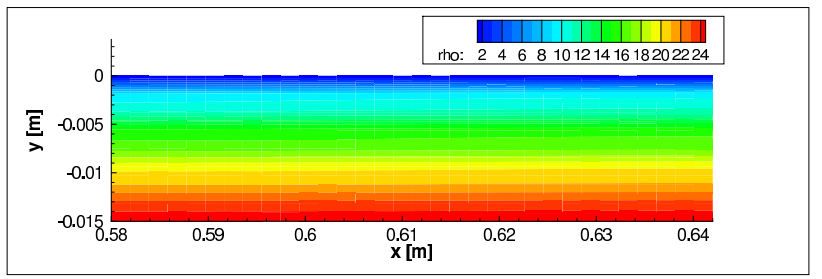

(c) $z=0.03 m$

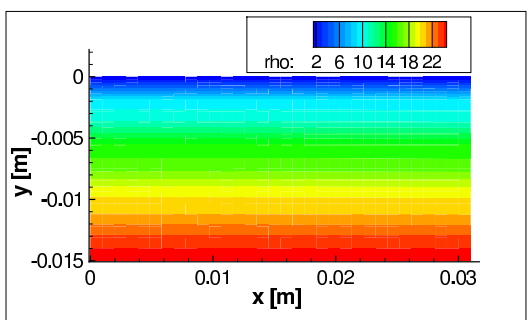

(b) $x=0.58 m$

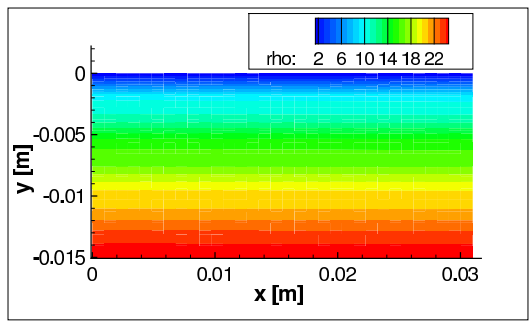

(d) $x=0.64 m$

Figure 22. Coolant density in the porous medium at different spanwise ( (a) and (c) ) and streamwise ( (b) and (d) ) positions.

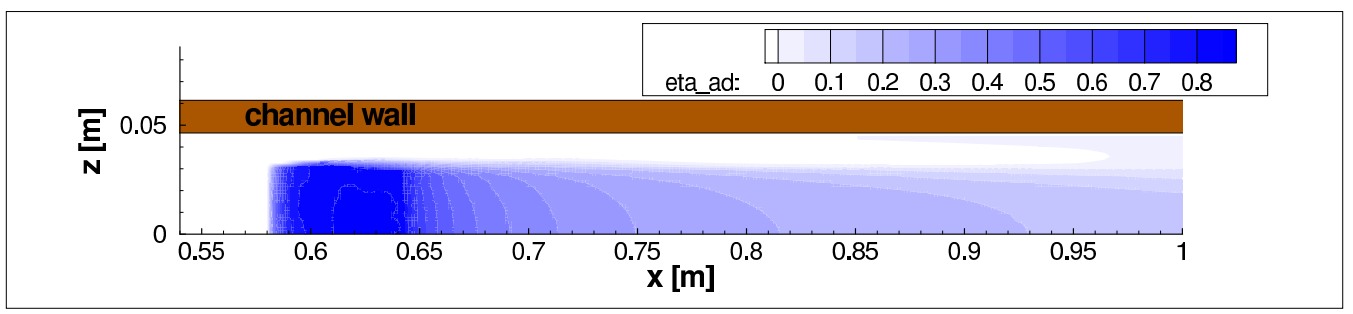

Figure 23. Cooling efficiency $\eta_{a d}$.

material, for $z=0.03 \mathrm{~m}$, the cooling efficiency is strongly reduced. Interestingly, the cooling efficiency on the centerline is higher compared to the two-dimensional results. The most probable reason is the influence of the channel walls narrows the cooled area. Therefore, the coolant is restricted to a smaller area which increases the cooling effect.

Finally, a quantitative comparison of infrared measurements [7] of the temperature on the surface of the porous material in the experiments by Langener [7] with the computational results is shown in Fig. 25. For both measurement and simulation, only the upper half of the porous medium is shown. Due to the developing coolant film, the surface is hotter at the leading edge than at the trailing edge. Furthermore, the temperature near all edges is increased, especially in the corners. There are two effects this could be attributed to. The side surfaces of the porous wall have been sealed with an epoxy slurry to avoid leaking coolant. This sealing might have entered some of the pores and led to a lower permeability close to the edges. This would result in lower local coolant mass fluxes and therefore higher temperatures. In addition, since the cooled porous material can become less hot than the surrounding channel walls heat conduction into the porous material can occur [7]. Of course, if one had estimates of the corresponding heat fluxes these could be easily incorporated in the simulations. The checkered structure results from the production process of the $\mathrm{C} / \mathrm{C}$ material. 


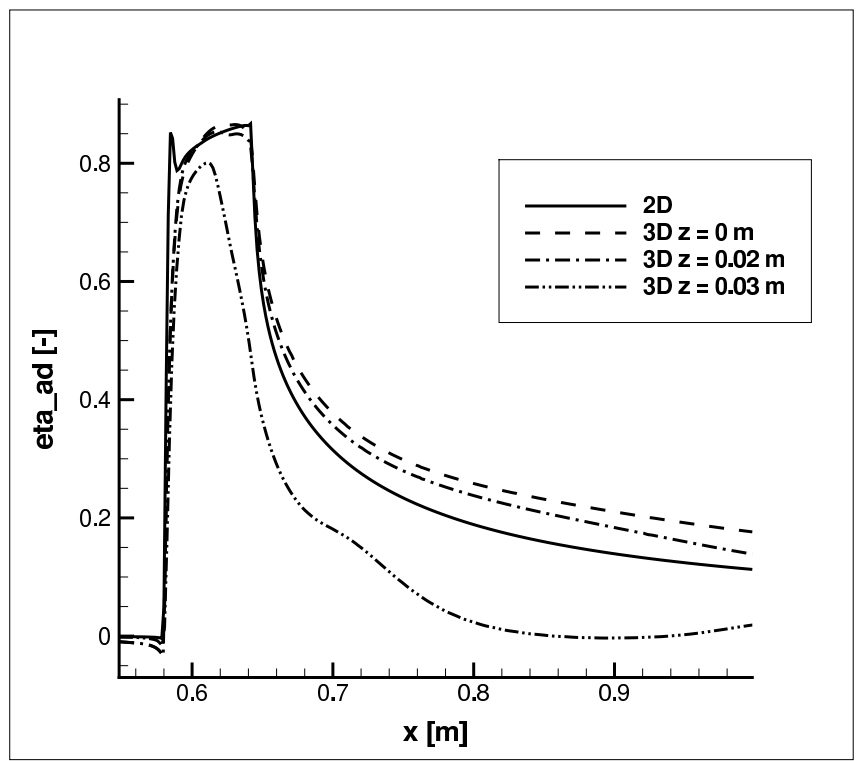

Figure 24. Cooling efficiency $\eta_{a d}$ : Comparison of two-dimensional and three-dimensional computations.

The reflection of the layers with different fiber orientation differs which results in these disturbances of the infrared picture.

Fig. 25(b) shows the surface temperature for the three-dimensional simulation. Again, the temperature at the leading edge is higher than at the trailing edge. Furthermore, it is slightly increased towards the sidewalls of the channel. This effect cannot be explained by the two causes given for the experiment before since the permeabilities are constant for the whole computational domain and no heat transfer from the surrounding channel walls are included in the boundary conditions. Therefore, this effect most likely results from the influences of the sidewalls of the channel on the hot gas flow.

The comparison of experimental and computational results shows an overall acceptable agreement. But there are certain significant deviations. These differences can be explained by undesirable but also unavoidable effects in the experiments which are not modeled in the simulations. Incorporating these effects would require additional information gained from experiments. Corresponding numerical computations would yield results that match experiments for a wider range of flow scenarios offering more detailed insight into the flow features and thermal behavior of both hot gas and porous medium. Conversely, simulations can quantify the influence of these undesirable effects, for example the impact of the channel sidewalls on the measurements. This leads to a better interpretation of the experimental results.

\section{CONCLUSION}

Advanced mathematical concepts are used to perform highly resolved numerical simulations of cooling gas injection through porous material. These reveal a detailed insight into small scale effects. This allows for reliable evaluation of active cooling techniques. Further investigations are needed 


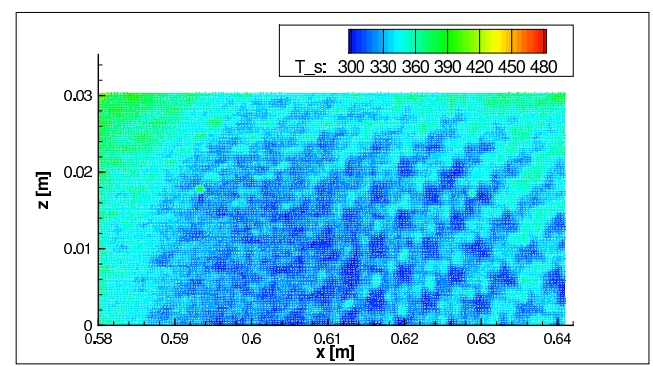

(a) Infrared thermography

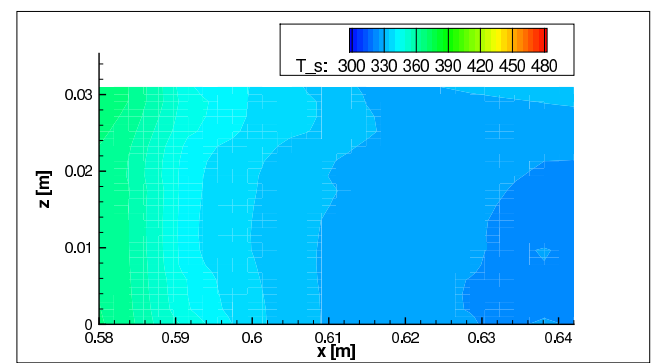

(b) Three-dimensional computation

Figure 25. Surface temperature of upper half of porous material: Infrared thermography (a) (courtesy of Langener [7]) and three-dimensional computation (b).

that incorporate enhanced techniques such as the usage of different coolants or micro-scale effects due to the roughness of the porous surface.

\subsection{Mathematical concept}

The numerical investigations confirm that the finite element solver presented in this paper is suitable for simulating porous media flow. Detailed insight into the distribution of temperature and flow in the porous material can be derived from the numerical results, where experimental results are again limited due to the small extent of the porous material. The placement of measurement devices into such a small regime is very difficult and their distance from each other is limited. Since the throughflow velocity in the porous medium is rather small, no stabilization of the numerical discretization is necessary. The comparison with experiments confirms that the porous media solver adequately reproduces the temperature distribution and the mass flow through the porous material. Nevertheless, there is a strong dependence on parameters like the heat exchange coefficients, which have yet to be determined by experiments.

We emphasize that common restrictive assumptions which are often used for modeling transpiration cooling are not assumed here. Therefore, the legitimacy of some of these assumptions could be reviewed. First of all, the coolant mass flux distribution is often prescribed. The results presented here show that both the coolant density and its Darcy velocity are almost constant in crosssections normal to the porous medium flow direction. Therefore, if high-quality measurements of the mass flux are available, this assumption can be justified. In contrast to this, one-dimensional modeling of the temperatures in the porous medium is a very restrictive assumption. The results presented here show a strong variation especially in streamwise direction. Finally, often the temperatures of fluid and solid part of the porous medium are assumed to be in equilibrium. Even considering that the results presented here are vague in respect to the heat transfer between solid and fluid due to the lack of exact measurements of the heat transfer coefficients, it is fair to say that they show a significant difference between both temperatures.

Coupling of porous medium flow to pure fluid flow is a field of research which lacks in both theoretical investigations and practical application of numerical tools. As stated in Section 1, to the authors knowledge, there has been no approach of performing a fully coupled simulation of transpiration cooling so far. The weak coupling using boundary conditions performed in this study leads to convergence and reasonable results. 


\subsection{Technological concept}

The numerical simulation of transpiration cooling by coupled porous media and pure compressible fluid flow have proven that this cooling technique can benefit from computational results as well. The results give detailed insight into the thermodynamic condition in the porous material. Furthermore, the influence of the injection on the turbulent channel flow can be observed. By investigating the influence of the channel walls on the computational results, the value and the assignability of the experimental results to the real application can be re-evaluated.

Compared with film cooling approaches using for example slots or holes the injection of a coolant through a porous material tends to promote more the cooling of the structure itself rather than the development of a film on the surface of the wall downstream from the injection. Furthermore, the area where the coolant is injected through the porous material can be large compared to slots or multiple boreholes. Therefore, blowing ratios are smaller and the interaction with the hot gas flow is weaker. Therefore, the coolant film inserted by the transpiration cooling approach is more uniform compared to the one produced by blowing through boreholes, for example.

\subsection{Future investigations}

In the present work only air is considered as cooling gas. With regard to an optimal cooling efficiency, different coolants should be investigated. This can have a major impact on the thickness of the cooling layer. The injection of different coolants through porous material into both subsonic and supersonic hot gas flows has been investigated experimentally by Langener [7]. In order to perform numerical simulations the air flow and the injected coolants have to be modeled as a mixture of perfect gases. For this purpose, the QUADFLOW solver is currently being extended, see Windisch [11].

In the experiments corresponding to the simulations in this work the porous material has been mounted into the wall in such a way that the carbon fibers are orthogonal to the porous media flow. This has the advantage that the heat conduction in wall-normal direction is reduced. In contrast to this, the pressure difference between hot gas flow and coolant reservoir which is necessary to reach a certain Darcy velocity in the porous material is higher. Experiments with a second probe, where the throughflow is parallel to the fibers, have been considered by Gotzen [34]. Due to differences in the production process for probes for orthogonal and parallel throughflow, mainly due to the necessary size of the probe in different directions, the material parameters differ and do not allow for a decision which direction should be preferred.

Future interest will be concerned also with the use of higher order turbulence models. Therefore, turbulence models as the Reynolds stress model or the Variational Multiscale Method are to be applied. The latter can be considered an advanced LES, see Koobus and Farhat [36]. Another suitable ansatz could be the coherent vortex structure (CVS) approach proposed by Farge and Schneider [37]. The quality of the results will benefit much more from the higher order turbulence modeling for more complicated and more realistic applications.

The physical modeling currently used for the porous media flow does not take into account friction in the velocity equation of the porous medium. This is sufficient in the interior of the domain, but may be not correct at the interface between porous medium and hot gas flow. Currently, looking from the hot gas flow, the interface to the porous material is modeled as a no-slip boundary, in 
tangential direction. But on a microscopic scale, this is only true concerning the solid part of the porous material. The hot gas flow will introduce a tangential shear stress to the fluid part of the porous material, which will lead to a thin layer at the interface with fluid inside the porous material moving in streamwise direction of the hot gas flow. But, as mentioned before, the physical modeling of such a boundary layer is not possible without introducing friction to the model for the porous medium flow. Therefore, only injection in wall-normal direction has been considered so far.

In accordance with other authors the assumption was made that the pressure is continuous across the interface between the flow domain and the porous material. Nield and Bejan [29] pointed out that this is in fact the case on the microscopic scale, but might not hold on the macroscopic scale. There can be a relevant pressure difference in a thin layer around the interface that cannot easily be resolved in numerical computations.

Without friction, the flow of the coolant through the porous material is quasi-one-dimensional. Therefore, the nonlinear equations for density and Darcy velocity could be solved in a onedimensional domain, leaving only the linear system for the two temperatures to be solved in two or three space dimensions. This would reduce computational costs.

Furthermore, the weakly coupled simulations in this work have been done without performing a fixed point iteration at the interface between the porous material and the hot gas flow. Stationary solutions are reached in both the hot gas flow and the porous medium and these solutions do not change after applying more coupling iterations. A more thorough investigation of the coupling process solving a fixed point problem will be necessary for instationary applications.

In order to further improve the model homogenization techniques might be deployed in the future to determine effective boundary conditions for porous medium injection. This will give the possibility to take small scale effects into account that are caused by the roughness of the porous medium surface which in turn cannot be directly resolved. For incompressible, laminar flow results are reported in [38].

\section{ACKNOWLEDGEMENT}

The authors would like to thank Prof. Dr. J. von Wolfersdorf and S. Schweikert, University of Stuttgart, and Dr. T. Langener, European Space Agency, for fruitful discussions on the modeling of porous medium flow and providing us with experimental data.

\section{REFERENCES}

1. N. Tramecourt, M. Masquelet, S. Menon, Large-Eddy Simulation of Unsteady Wall Heat Transfer in a High Pressure Combustion Chamber, 41st AIAA/ASME/SAE/ASEE Joint Propulsion Conference \& Exhibit, Tucson, Arizona, 117, 2005.

2. F. Bramkamp, Ph. Lamby, S. Müller, An adaptive multiscale finite volume solver for unsteady and steady state flow computations, J. Comp. Phys., 197 (2), 460-490 (2004).

3. S. Müller, Adaptive Multiscale Schemes for Conservation Laws, Lecture Notes on Computational Science and Engineering, 27, first ed., Springer Verlag (2003).

4. Ph. Lamby, Parametric Multi-Block Grid Generation and Application to Adaptive Flow Simulations, Diss. RWTH Aachen (2007).

5. M. Bachmann, K. Brix, S. Melian, S. Müller, Adaptive Multiresolution Methods: Practical Issues on Data Structures, Implementation and Parallelization, in: Summer school on multiresolution and adaptive mesh refinement methods, E. Cancès, V. Louvet, M. Massot (eds.), ESAIM: Proceedings, Vol. 34, 151-183 (2011). 
6. F.R. Menter, Two-Equation Eddy-Viscosity Turbulence Models for Engineering Applications, AIAA J., 32, 15981605 (1994).

7. T. Langener, A Contribution to Transpiration Cooling for Aerospace Applications Using CMC Walls, Diss. Univ. Stuttgart (2011).

8. W. Bangerth, R. Hartmann, G. Kanschat, deal.II - a general-purpose object-oriented finite element library, ACM Trans. Math. Softw., 33(4), 24/1-24/27 (2007).

9. W. Dahmen, T. Gotzen, S. Melian, S. Müller, Numerical simulation of cooling gas injection using adaptive multiresolution techniques, Comp. and Fluids 71, 65-82 (2013).

10. V. Gerber, Modellierung und Simulation von Strömungen in porösen Medien, Bachelor thesis, RWTH Aachen (2012).

11. Ch. Windisch, B. Reinartz, S. Müller, Numerical Simulation of Coolant Variation in Laminar Supersonic Film Cooling, 50th AIAA Aerospace Sciences Meeting, 9-12 January 2012, Nashville, Tennessee, AIAA-2012-949.

12. A. Herbertz, Numerische Leistungsanalyse von Triebwerksauslegungen mit transpirativ gekühlter keramischer Raketenbrennkammer, DGLR Jahrestagung, 2003-103, November 2003.

13. D. Greuel, A. Herbertz, J. Haidn, M. Ortelt, H. Hald, Transpiration Cooling Applied to C/C Liners of Cryogenic Liquid Rocket Engines, 40th AIAA/ASME/SAE/ASEE Joint Propulsion Conference and Exhibit, 11-14 July 2004, AIAA 2004-3682.

14. T. Langener, J. von Wolfersdorf, J. Steelant, Experimental investigations on transpiration cooling for Scramjet applications using different coolants, AIAA J. 49(7), 1409-1419 (2011).

15. W. Kays, M. Crawford, B. Weigand, Convective Heat and Mass Transfer, 4th ed., McGraw-Hill, New York (2005).

16. T. Langener, J. von Wolfersdorf, M. Selzer, H. Hald, Experimental investigations of transpiration cooling applied to C/C material, Intl. J. Thermal Sc. 54, 70-81 (2012).

17. M. Selzer, T. Langener, H. Hald, J. von Wolfersdorf, Production and Characterization of Porous C/C Material, In: N.A. Adams, R. Radespiel, T. Sattelmayer, W. Schröder, B. Weigand (Eds.), Annual Report SFB TRR40 2009 , München, 75-85 (2009).

18. S. Gulli, L. Maddalena, S. Hosder, Investigation of Transpiration Cooling Effectiveness for Air-Breathing Hypersonic Vehicles, 17th AIAA International Space Planes and Hypersonic Systems and Technologies Conference, 11 - 14 April 2011, San Francisco, California, AIAA 2011-2253 (2011).

19. D. E. Glass, A. D. Diley, H. N. Kelly, Numerical Analysis of Convection/Transpiration Cooling, NASA TM-1999209828, NASA Langley Research Center, Hampton, VA (1999).

20. L. R. Tully, Numerical Investigations of Transpiration Cooled Rocket Injectors, Master Thesis, University of Florida (2005).

21. L. Mathelin, F. Bataille, A. Lallemand, Blowing models for cooling surfaces, Int. J. Therm. Sci. 40, 969-980 (2001).

22. T. Langener, J. von Wolfersdorf, F. Cheuret, J. Steelant, M. Kuhn, Experimental and Numerical Study on Transpiration Cooling with Supersonic Flow, 19th ISABE Conference, Montral, Kanada (2009).

23. F. Cheuret, J. Steelant, T. Langener, J. von Wolfersdorf, Simulations on Transpiration Cooling for Supersonic Flow, Proceedings of the CEAS 2009 European Air and Space Conference, Manchester, UK, 2009.

24. J. Steelant, ATLLAS: Aero-Thermal Loaded Material Investigations for High-Speed Vehicles, 15th AIAA International Space Planes and Hypersonic Systems and Technologies Conference, Dayton, OH, 2008, AIAA2008-2582.

25. N. Gascoin, L. Romagnosi, I. Fedioun, G. Fau, P. Gillard, J. Steelant, Pyrolysis in Porous Media: Numerical Analysis and Comparison to Experiments, 18th AIAA/3AF International Space Planes and Hypersonic Systems and Technologies Conference, 24 - 28 September 2012, Tours, France, AIAA 2012-5848.

26. L. Romagnosi, N. Gascoin, E. El-Tabach, C. Strozzi, I. Fedioun, J. Steelant, Pyrolysis in Porous Media: Numerical model and parametric study, 18th AIAA/3AF International Space Planes and Hypersonic Systems and Technologies Conference, 24 - 28 September 2012, Tours, France, AIAA 2012-5876.

27. V. Hannemann, Numerical investigation of an effusion cooled thermal protection material, Intl. Conf. Comp. Fluid Dyn. 4, Ghent, Belgium, 2006.

28. F. Bramkamp, Unstructured h-Adaptive Finite-Volume Schemes for Compressible Viscous Fluid Flow, Diss. RWTH Aachen (2003).

29. D.A. Nield, A. Bejan, Convection in Porous Media. Springer, 3rd edition, USA (2006).

30. T.P. Fries, H.G. Matthies, A Review of Petrov-Galerkin Stabilization Approaches and an Extension to Meshfree Methods, Informatikbericht-Nr. 2004-01, Technische Universität Braunschweig, Brunswick, Germany (2004).

31. H. Elman, D. Silvester, A. Wathen, Finite Elements and Fast Iterative Solvers: With Applications in Incompressible Fluid Dynamics, Oxford University Press (2005).

32. T. A. Davis, I. S. Duff, An unsymmetric-pattern multifrontal method for sparse LU factorization, SIAM J. Matrix Anal. Appl., 18(1), 140-158 (1997). 
33. T. A. Davis, A column pre-ordering strategy for the unsymmetric-pattern multifrontal method, ACM Trans. Math. Softw., 30(2), 165-195 (2004).

34. T. Gotzen, Numerical investigation of film and transpiration cooling, Diss. RWTH Aachen (2013).

35. T.J.R. Hughes, The Finite Element Method: Linear Static and Dynamic Finite Element Analysis, Dover Publications, 2000.

36. B. Koobus, Ch. Farhat, A variational multiscale method for the large eddy simulation of compressible turbulent flows on unstructured meshes - application to vortex shedding, Comp. Meth. Appl. Mech. and Eng., 193, 13671383 (2004).

37. M. Farge, K. Schneider, Coherent Vortex simulation (CVS), a semi-deterministic turbulence model using wavelets, Flow, Turb. and Comb., 66(4), 393-426 (2001).

38. W. Jäger, A. Mikelic, On the roughness-induced effective boundary conditions for a viscous flow, J. Diff. Eq. 170, 96-122 (2001) 